\title{
Color Representations of Ising Models
}

\author{
Malin P. Forsström ${ }^{1}$ \\ Received: 5 August 2020 / Revised: 6 October 2020 / Accepted: 22 October 2020 / \\ Published online: 9 November 2020 \\ (c) The Author(s) 2020
}

\begin{abstract}
In Steif and Tykesson (J Prob 16:899-955, 2019), the authors introduced the so-called general divide and color models. One of the best-known examples of such a model is the Ising model with external field $h=0$, which has a color representation given by the random cluster model. In this paper, we give necessary and sufficient conditions for this color representation to be unique. We also show that if one considers the Ising model on a complete graph, then for many $h>0$, there is no color representation. This shows, in particular, that any generalization of the random cluster model which provides color representations of Ising models with external fields cannot, in general, be a generalized divide and color model. Furthermore, we show that there can be at most finitely many $\beta>0$ at which the random cluster model can be continuously extended to a color representation for $h \neq 0$.
\end{abstract}

Mathematics Subject Classification (2020) 60G99 • 60K35

\section{Introduction}

A simple mechanism for constructing random variables with a positive dependency structure is the so-called generalized divide and color model. This model was first introduced in [12], but similar constructions had already arisen in many different contexts.

Definition 1.1 Let $S$ be a finite set. A $\{0,1\}^{S}$-valued random variable $X:=\left(X_{i}\right)_{i \in S}$ is called a generalized divide and color model if $X$ can be generated as follows.

1. Choose a random partition $\pi$ of $S$ according to some arbitrary distribution $\mu$.

2. Let $\pi_{1}, \ldots, \pi_{m}$ be the partition elements of $\pi$. Independently for each $i \in$ $\{1,2, \ldots, m\}$, pick a "color" $c_{i} \sim(1-p) \delta_{0}+p \delta_{1}$, and assign all the elements in $\pi_{i}$ the color $c_{i}$ by letting $X_{j}=c_{i}$ for all $j \in \pi_{i}$.

\footnotetext{
$凶 \quad$ Malin P. Forsström malinpf@kth.se

1 Department of Mathematics, KTH Royal Institute of Technology, 10044 Stockholm, Sweden
} 
The final $\{0,1\}$-valued process $X$ is called the generalized divide and color model associated to $\mu$ and $p$, and we say that $\mu$ is a color representation of $X$.

As detailed in [12], many processes in probability theory are generalized divide and color models, one of the most prominent examples being the Ising model with no external field. To define this model, let $G=(V, E)$ be a finite connected graph with vertex set $V$ and edge set $E$. We say that a random vector $X=\left(\sigma_{i}\right)_{i \in V} \in\{0,1\}^{V}$ is an Ising model on $G$ with interaction parameter $\beta>0$ and external field $h \in \mathbb{R}$ if $X$ has probability density function $v_{G, \beta, h}$ proportional to

$$
\exp \left(\beta \sum_{\{i, j\} \in E}\left(\mathbb{1}_{\sigma_{i}=\sigma_{j}}-\mathbb{1}_{\sigma_{i} \neq \sigma_{j}}\right)+h \sum_{i \in V}\left(\mathbb{1}_{\sigma_{i}=1}-\mathbb{1}_{\sigma_{i}=0}\right)\right)
$$

The parameter $\beta$ will be referred to as the interaction parameter and the parameter $h$ as the strength of the external field. We will write $X^{G, \beta, h}$ to denote a random variable with $X^{G, \beta, h} \sim v_{G, \beta, h}$. It is well known that the Ising model has a color representation when $h=0$ given by the random cluster model. To define the random cluster model associated with the Ising model we first, for $G=(V, E)$ and $w \in\{0,1\}^{E}$, define $E_{w}:=\left\{e \in E: w_{e}=1\right\}$ and note that this defines a partition $\pi[w]$ of $V$, where $v, v^{\prime} \in V$ are in the same partition element of $\pi[w]$ if and only if they are in the same connected component of the graph $\left(V, E_{w}\right)$. Let $\|\pi[w]\|$ be the number of partition elements of $\pi[w]$ and let $\mathcal{B}_{V}$ denote the set of partitions of $V$. For $r \in(0,1)$ and $q \geq 0$, the random cluster model $\mu_{G, r, q}$ is defined by

$$
\mu_{G, r, q}\left(\pi^{\prime}\right)=\frac{1}{Z_{G, r, q}^{\prime}} \sum_{\substack{w \in\{0,1\}^{E} \\ \pi[w]=\pi^{\prime}}}\left[\prod_{e \in E} r^{w(e)}(1-r)^{1-w(e)}\right] q^{\|\pi[w]\|}, \quad \pi^{\prime} \in \mathcal{B}_{V}
$$

where $Z_{G, r, q}^{\prime}$ is a normalizing constant ensuring that this is a probability measure. It is well known (see, e.g., [10]) that if one sets $r=1-\exp (-2 \beta), q=2$ and $p=1 / 2$, then $\mu_{G, r, q}$ is a color representation of $X^{\beta, 0}$. To simplify notation, we will write $\mu_{G, r}:=\mu_{G, r, 2}$.

Since many properties of the Ising model with $h=0$ have been understood by using a color representation (given by the random cluster model, see, e.g., $[1,6,10]$ ), it is natural to ask if there is a color representation also when $h>0$. Moreover, Theorems 1.2 and 1.4 in [8], which state that a random coloring of a set can have more than one color representation, motivates asking whether there are any color representation when $h=0$ which is different from the random cluster model. The main objective of this paper is to provide partial answers to these questions by investigating how generalized divide and color models relate to some Ising models, both in the presence and absence of an external field.

In order to be able to present these results, we will need some additional notation. Let $S$ be a finite set. For any measurable space $(S, \sigma(S))$, we let $\mathcal{P}(S)$ denote the set of probability measures on $(S, \sigma(S))$. When $S=\{0,1\}^{T}$ for some finite set $T$, then we always consider the discrete $\sigma$-algebra, i.e., we let $\sigma\left(\{0,1\}^{T}\right)$ be the set of all subsets of $\{0,1\}^{T}$. Recall the we let $\mathcal{B}_{S}$ denote the set of partitions of $S$. If $\pi \in \mathcal{B}_{S}$ 
and $T \subseteq S$, we let $\left.\pi\right|_{T}$ denote the partition of $T$ induced from $\pi$ in the natural way. On $\mathcal{B}_{S}$ we consider the $\sigma$-algebra $\sigma\left(\mathcal{B}_{S}\right)$ generated by $\left\{\left.\pi\right|_{T}\right\}_{T \subseteq S, \pi \in \mathcal{B}_{S}}$. In analogy with [12], we let $\operatorname{RER}_{S}$ denote the set of all probability measures on $\left(\mathcal{B}_{S}, \sigma\left(\mathcal{B}_{\mathcal{S}}\right)\right.$ ) (RER stands for random equivalence relation). For a graph $G$ with vertex set $V$ and edge set $E$, we let $\operatorname{RER}_{V}^{G}$ denote the set of probability measures $\mu \in \mathrm{RER}_{V}$ which has support only on partitions $\pi \in \mathcal{B}_{V}$ whose partition elements induce connected subgraphs of $G$. For each $p \in(0,1)$, we now introduce the mapping $\Phi_{p}$ from $\operatorname{RER}_{S}$ to the set of probability measures on $\{0,1\}^{S}$ as follows. Let $\mu \in \operatorname{RER}_{S}$. Pick $\pi$ according to $\mu$. Let $\pi_{1}, \pi_{2}, \ldots, \pi_{m}$ be the partition elements of $\pi$. Independently for each $i \in\{1,2, \ldots, m\}$, pick $c_{i} \sim(1-p) \delta_{0}+p \delta_{1}$ and let $X_{j}=c_{i}$ for all $j \in \pi_{i}$. This yields a random vector $X=\left(X_{i}\right)_{i \in S}$ whose distribution will be denoted by $\Phi_{p}(\mu)$. The random vector $X$ will be referred to as a generalized divide and color model, and the measure $\mu$ will be referred to as a color representation of $X$ or $\Phi_{p}(\mu)$. Note that $\Phi_{p}: \mathcal{B}_{S} \rightarrow \mathcal{P}\left(\{0,1\}^{S}\right)$. We will say that a probability measure $v \in \mathcal{P}\left(\{0,1\}^{S}\right)$ has a color representation if there is a measure $\mu \in \operatorname{RER}_{S}$ and $p \in(0,1)$ such that $v=\Phi_{p}(\mu)$. For $v \in \mathcal{P}(S)$, we let $\Phi^{-1}(v):=\left\{\mu \in R E R_{S}: \Phi_{p}(\mu)=v\right\}$. Then, $v$ has a color representation if and only iff there is $p \in(0,1)$ such that $\Phi_{p}^{-1}(v)$ is non-empty. By Theorems 1.2 and 1.4 in [8], $\Phi_{p}^{-1}(\nu)$ can be non-empty if and only if the one-dimensional marginals of $v$ are all equal to $(1-p) \delta_{0}+p \delta_{1}$. From this it immediately follows that for any graph $G$ and any $\beta>0$, we have $\Phi_{p}^{-1}\left(v_{G, \beta, 0}\right)=\emptyset$ whenever $p \neq 1-e^{-2 \beta}$.

Our first result is the following theorem, which states that for any finite graph $G$ and any $\beta>0, X^{G, \beta, 0}$ has at least two distinct color representations.

Theorem 1.2 Let $n \in \mathbb{N}$ and let $G$ be a connected graph with $n \geq 3$ vertices. Further, let $\beta>0$. Then, there are at least two distinct probability measures $\mu, \mu^{\prime} \in R E R_{V(G)}$ such that $\Phi_{1 / 2}(\mu)=\Phi_{1 / 2}\left(\mu^{\prime}\right)=v_{G, \beta, 0}$. Furthermore, if $G$ is not a tree, then there are at least two distinct probability measures $\mu, \mu^{\prime} \in R E R_{V(G)}^{G}$ such that $\Phi_{1 / 2}(\mu)=$ $\Phi_{1 / 2}\left(\mu^{\prime}\right)=v_{G, \beta, 0}$.

We remark that if a graph $G$ has only one or two vertices and $\beta>0$, then it is known from Theorem 2.1 in [12] (see also Theorems 1.2 and 1.4 in [8]) that $\Phi_{1 / 2}^{-1}\left(v_{G, \beta, 0}\right)=$ $\left\{\mu_{G, 1-\exp (-2 \beta)}\right\}$. In other words, when $h=0$, the Ising model $X^{G, \beta, h}$ has a unique color representation, given by the random cluster model $\mu_{G, 1-\exp (-2 \beta)}$. To get an intuition for what should happen when $h>0$, we first look at a few toy examples. One of the simplest such examples is the Ising model on a complete graph with three vertices. The following result was also included as Remark 7.8(iii) in [12] and in [8] as Corollary 1.8.

Proposition 1.3 Let $G$ be the complete graph on three vertices. Let $\beta>0$ be fixed. For each $h>0$, let $p_{h} \in(0,1)$ be such that the marginal distributions of $X^{G, \beta, h}$ are given by $\left(1-p_{h}\right) \delta_{0}+p_{h} \delta_{1}$. Then, the following holds.

(i) For each $h>0$, we have $\left|\Phi_{p_{h}}^{-1}\left(v_{G, \beta, h}\right)\right|=1$, i.e., $X^{G, \beta, h}$ has a unique color representation for any $h>0$.

(ii) For each $h>0$, let $\mu_{h}$ be defined by $\left\{\mu_{h}\right\}=\Phi_{p(\beta, h)}^{-1}\left(v_{G, \beta, h}\right)$. Then, $\mu_{0}(\pi):=$ $\lim _{h \rightarrow 0} \mu_{h}(\pi)$ exists for all $\pi \in \mathcal{B}_{V(G)}$, and $\Phi_{1 / 2}\left(\mu_{0}\right)=v_{G, \beta, 0}$. However, $\mu_{0} \neq$ $\mu_{G, 1-e^{-2 \beta} \text {. }}$ 
Interestingly, if we increase the number of vertices in the underlying graph by one, the picture immediately becomes more complicated.

Proposition 1.4 Let $G$ be the complete graph on four vertices. Let $\beta>0$ be fixed. For each $h>0$, let $p_{h} \in(0,1)$ be such that the marginal distributions of $X^{G, \beta, h}$ are given by $\left(1-p_{h}\right) \delta_{0}+p_{h} \delta_{1}$. To simplify notation, set $x:=e^{2 \beta}$ and $y_{h}:=e^{2 h}$. Then, $X^{G, \beta, h}$ has a color representation if and only if

$$
\begin{aligned}
x^{5} & +3 x^{2} y_{h}+4 x y_{h}^{2}-2 x^{3} y_{h}^{2}+x^{5} y_{h}^{2}-3 y_{h}^{3}+7 x^{2} y_{h}^{3}-x^{4} y_{h}^{3}-x^{6} y_{h}^{3} \\
& +4 x y_{h}^{4}-2 x^{3} y_{h}^{4}+x^{5} y_{h}^{4}+3 x^{2} y_{h}^{5}+x^{5} y_{h}^{6} \geq 0 .
\end{aligned}
$$

In particular, if we let $\beta_{0}:=\log (2+\sqrt{3}) / 2$, then the following holds.

(i) If $\beta<\beta_{0}$, then $X^{G, \beta, h}$ has a color representation for all sufficiently small $h>0$,

(ii) If $\beta>\beta_{0}$, then $X^{G, \beta, h}$ has no color representation for any sufficiently small $h>0$.

Moreover, there is no decreasing sequence $h_{1}, h_{2}, \ldots$ with $\lim _{n \rightarrow \infty} h_{n}=0$ and $\mu_{h_{n}} \in$ $\Phi_{p_{h_{n}}}^{-1}\left(v_{G, \beta, h}\right)$ such that $\lim _{n \rightarrow \infty} \mu_{h_{n}}(\pi)=\mu_{G, 1-e^{-2 \beta}}(\pi)$ for all $\pi \in \mathcal{B}_{V(G)}$. In other words, the random cluster model does not arise as a subsequential limit of color representations of $X^{G, \beta, h}$ as $h \rightarrow 0$, for any $\beta>0$.

Interestingly, this already shows that there are graphs $G$ and parameters $\beta, h>0$ such that the corresponding Ising model $X^{G, \beta, h}$ does not have any color representations.

The proof of Proposition 1.4 can in principle be extended directly to complete graphs with more than four vertices, but it quickly becomes computationally heavy and the analogues of (1) become quite involved (see Remark 4.1 for the analogue expression for a complete graph on five vertices). In Fig. 1, we draw the set of all pairs $(\beta, h) \in \mathbb{R}_{+}^{2}$ which satisfies the inequality in (1) together with the corresponding set for a complete graph on five vertices.

Figure 1, together with the previous two propositions, suggests that the following conjectures should hold for all complete graphs $G$ on at least four vertices.

I. If $\beta>0$ is sufficiently small, then $X^{G, \beta, h}$ has a color representation for all $h \in \mathbb{R}$.

II. For each $\beta>0, X^{G, \beta, h}$ has a color representations for all sufficiently large $h \in \mathbb{R}$.

III. If $\beta$ is sufficiently large, then $X^{G, \beta, h}$ has no color representation for any sufficiently small $h>0$.

IV. If $\beta, h>0$ and $X^{G, \beta, h}$ has a color representation, then so does $X^{G, \beta^{\prime}, h}$ and $X^{G, \beta, h^{\prime}}$ for all $h^{\prime}>h$ and $\beta^{\prime} \in(0, \beta)$.

V. The random cluster model corresponding to $X^{G, \beta, 0}$ does not arise as a subsequential limit of color representations of $X^{G, \beta, h}$ as $h \rightarrow 0$.

Our next result concerns the last of these conjectures.

Theorem 1.5 Let $n \in \mathbb{N}$ and let $G$ be a connected and vertex-transitive graph with $n$ vertices. For each $\beta \geq 0$ and $h>0$, let $p_{\beta, h} \in(0,1)$ be such that the marginal distributions of $X^{G, \beta, h}$ are given by $\left(1-p_{\beta, h}\right) \delta_{0}+p_{\beta, h} \delta_{1}$. Then, there is a set $B \subseteq \mathbb{R}_{+}$ with $|B| \leq n(n-1)$ such that for all $\beta \in \mathbb{R}_{+} \backslash B$, all sequences $h_{1}>h_{2}>\ldots$ with $\lim _{m \rightarrow \infty} h_{m}=0$ and all sequences $\mu_{m} \in \Phi_{p_{\beta, h_{m}}}^{-1}\left(v_{G, \beta, h}\right)$, there is a partition $\pi \in \mathcal{B}_{V}$ 
Fig. 1 The sets of all $(\beta, h) \in \mathbb{R}_{+}^{2}$ which are such that the Ising model $X^{G, \beta, h}$, for $G$ being a complete graph on four vertices (red) and five vertices (black) respectively, has at least one color representation (see Proposition 1.4 and Remark 4.1).

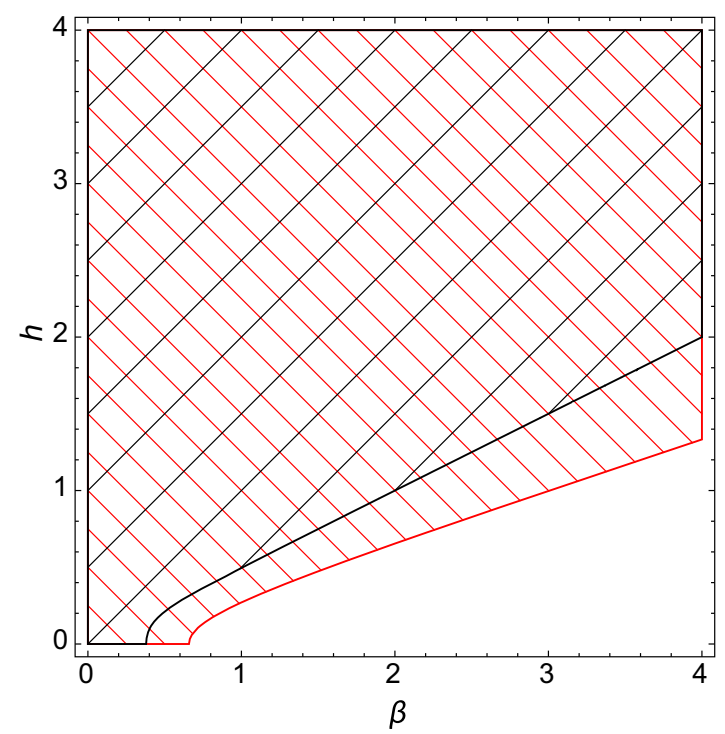

such that $\lim _{m \rightarrow \infty} \mu_{m}(\pi) \neq \mu_{G, 1-e^{-2 \beta}}(\pi)$. In other words, the random cluster model on $G$ can arise as a subsequential limit when $h \rightarrow 0$ of color representations of $X^{G, \beta, h}$ for at most $n(n-1)$ different values of $\beta$.

Interestingly, Theorem 1.5 does not require $n$ to be large. The set of exceptional values for $\beta$ where the random cluster model could arise as a limit is a consequence of the proof strategy used, and could possibly be shown to be empty by using a different proof.

Our next result shows that the third of the conjectures above is true when $n$ is sufficiently large.

Theorem 1.6 Let $n \in \mathbb{N}$ and let $G$ be the complete graph on $n$ vertices. Further, let $\hat{\beta}>0$ and $\beta:=\hat{\beta} / n$. If $\hat{\beta} \geq \hat{\beta}_{c}=1$ and $n$ is sufficiently large, then $X^{G, \beta, h}$ has no color representation for any sufficiently small $h>0$.

As a consequence of this theorem, the fifth conjecture above is true when $\beta>1 / n$ and $n$ is sufficiently large.

Our last result gives a partial answer to the second conjecture.

Theorem 1.7 Let $n \in \mathbb{N}$ and let $G$ be the complete graph on $n$ vertices. Let $h>0$ and $\beta=\beta(h)$ be such that $(n-1) \beta(h)<h$. Then, $X^{G, \beta(h), h}$ has a color representation for all sufficiently large $h$.

Simulations suggest that the previous result should be possible to extend to $\beta(h)<h$. This is a much stronger statement, especially for large $n$, and would require a different proof strategy. The assumption that $(n-1) \beta(h) \leq h$, made in Theorem 1.7, is, however, a quite natural condition, since this exactly corresponds to that $v_{G, \beta, h}\left(1^{S} 0^{[n] \backslash S}\right)$ is decreasing in $|S|$, for $S \subseteq[n]$ (here $1^{S} 0^{[n] \backslash S}$ denotes the binary string $x \in\{0,1\}^{n}$ with $x(i)=1$ when $i \in S$ and $x(i)=0$ when $\left.i \notin S\right)$. 
The rest of this paper will be structured as follows. In Sect. 2, we give the background and definitions needed for the rest of the paper. In Sect. 3, we give a proof of Theorem 1.2. In Sect. 4, we give proofs of Propositions 1.3 and 1.4 and also discuss what happens when $G$ is the complete graph on five vertices. Next, in Sect. 5, we prove Theorems 1.5 and 1.6, and in Sect. 6, we give a proof of Theorem 1.7. Finally, in Sect. 7, we state and prove a few technical lemmas which are used throughout the paper.

\section{Background and Notation}

The main purpose of this section is to give definitions of the notation used throughout the paper, as well as some more background to the questions studied.

\subsection{The Original Divide and Color Model}

When the generalized divide and color model was introduced in [12], it was introduced as a generalization of the so-called divide and color model, first defined by Häggström in [11]. To define this family of models, let $G$ be a finite graph with vertex set $V$ and edge set $E$. Let $r \in(0,1)$, and let $\lambda$ be a finitely supported probability measure on $\mathbb{R}$. The divide and color model $X=\left(X_{v}\right)_{v \in V}$ (associated to $r$ and $\lambda$ ) is the random coloring of $V$ obtained as follows.

1. Pick $\pi \sim \mu_{G, r, 1}$.

2. Let $\pi_{1}, \ldots, \pi_{m}$ be the partition elements of $\pi$. Independently for each $i \in$ $\{1,2, \ldots, m\}$, pick $c_{i} \sim \lambda$, and assign all the vertices $v \in \pi_{i}$ the color $c_{i}$ by letting $X_{v}=c_{i}$ for all $v \in \pi_{i}$

Note that if $\lambda=(1-p) \delta_{0}+p \delta_{1}$, then $X \sim \Phi_{p}\left(\mu_{G, r, 1}\right)$.

Since its introduction in [11], properties of the divide and color model have been studied in several papers, including, e.g., [3,4,9]. Several closely related models, which all in some way generalize the divide and color model by considering more general measures $\lambda$, have also been considered (see, e.g., [2]).

We stress that this is not the model we discuss in this paper. To avoid confusion between the divide and color model and the generalized divide and color model, we will usually talk about color representations rather than generalized divide and color models.

\subsection{Generalizations of the Coupling Between the Ising Model and the Random Cluster Model}

When $h>0$, there is a generalization of the random cluster model (see, e.g., [5]) from which the Ising model can be obtained by independently assigning colors to different partition elements. This model has been shown to have properties which can be used in similar ways as analogue properties of the random cluster model. However, since this model uses different color probabilities for different partition elements, it is not a generalized divide and color models. On the other hand, Proposition 1.4 
shows that there are graphs $G$ and parameters $\beta, h>0$ such that $X^{G, \beta, h}$ has no color representation. This motivates considering less restrictive generalizations of the random cluster model, such as the one given in [5].

\subsection{General Notation}

Let $\mathbb{1}$ denote the indicator function, and for each $n \in \mathbb{N}$, define $[n]:=\{1,2, \ldots, n\}$.

When $G$ is a graph, we will let $V(G)$ denote the set of vertices of $G$ and $E(G)$ denote its set of edges. For each graph $G$ with $|V(G)|=n$, we assume that a bijection from $V(G)$ to $[n]$ is fixed, and in this way identify binary strings $\sigma \in\{0,1\}^{V(G)}$ with the corresponding binary strings in $\{0,1\}^{n}$. The complete graph on $n$ vertices will be denoted by $K_{n}$.

For all finite sets $S$, disjoint sets $T, T^{\prime} \subseteq S$ and $\sigma=\left(\sigma_{i}\right)_{i \in S} \in\{0,1\}^{S}$, we now make the following definitions. Let $\left.\sigma\right|_{T}$ denote the restriction of $\sigma$ to $T$. Write $\left.\sigma\right|_{T} \equiv 1$ if $\sigma_{i}=1$ for all $i \in T$, and analogously write $\left.\sigma\right|_{T} \equiv 0$ if $\sigma_{i}=0$ for all $i \in T$. We let $1^{T} 0^{T^{\prime}}$ denote the unique binary string $\sigma \in\{0,1\}^{T \cup T^{\prime}}$ with $\left.\sigma\right|_{T} \equiv 1$ and $\left.\sigma\right|_{T^{\prime}} \equiv 0$. Whenever $v$ is a signed measure on $\left(\{0,1\}^{S}, \sigma\left(\{0,1\}^{S}\right)\right.$, we write $\nu\left(1^{T}\right):=\sum_{T^{\prime} \subseteq[n] \backslash T} v\left(1^{T \cup T^{\prime}} 0^{S \backslash\left(T \cup T^{\prime}\right)}\right)$. We let $\|\sigma\|:=\sum_{i \in S} \sigma_{i}$ and define $\chi_{T}(\sigma):=$ $\prod_{i \in T}(-1)^{\mathbb{1}_{\sigma_{i}=0}}=(-1)^{n-\|\sigma\|}$.

We now give some notation for working with set partitions. To this end, recall that when $S$ is a finite set we let $\mathcal{B}_{S}$ denote the set of partitions of $S$. If $S=[n]$ for some $n \in \mathbb{N}$, we let $\mathcal{B}_{n}:=\mathcal{B}_{[n]}$. If $\pi \in \mathcal{B}_{S}$ has partition elements $\pi_{1}, \pi_{2}, \ldots, \pi_{m}$, we write $\pi=\left(\pi_{1}, \ldots, \pi_{m}\right)$. Now assume that a finite set $S$, a partition $\pi \in \mathcal{B}_{S}$ and a binary string $\sigma \in\{0,1\}^{S}$ are given. We write $\pi \triangleleft \sigma$ if $\sigma$ is constant on the partition elements of $\pi$. If $\pi \triangleleft \sigma, \pi_{i}$ is a partition element of $\pi$ and $j \in \pi_{i}$, we write $\sigma_{\pi_{i}}:=\sigma_{j}$. Note that this function is well defined exactly when $\pi \triangleleft \sigma$. Next, we let $\|\pi\|$ denote the number of partition elements of $\pi$. Combining these notations, if $\pi \triangleleft \sigma$ then we let $\|\sigma\|_{\pi}:=\sum_{i=1}^{\|\pi\|} \sigma_{\pi_{i}}$. If $T \subseteq S$, we write $\left.\pi\right|_{T}$ to denote the restriction of the $\pi$ to the set $T$ (so that $\left.\pi\right|_{T} \in \mathcal{B}_{T}$ ). If $\mu$ is a signed measure on $\left(\{0,1\}^{S}, \sigma\left(\{0,1\}^{S}\right)\right), T \subseteq S$ and $\pi \in \mathcal{B}_{T}$, we let $\left.\mu\right|_{T}(\pi):=\mu\left(\left\{\pi^{\prime} \in \mathcal{B}_{\mathcal{S}}:\left.\pi^{\prime}\right|_{T}=\pi\right\}\right)$. If $\pi^{\prime}, \pi^{\prime \prime} \in \mathcal{B}_{S}$, then we write $\pi^{\prime} \triangleleft \pi^{\prime \prime}$ if for each partition element of $\pi^{\prime}$ is a subset of some partition element of $\pi^{\prime \prime}$.

We let $S_{n}$ denote the set of all permutations of $[n] . S_{n}$ acts naturally on $\mathcal{B}_{n}$ by permuting the elements in $[n]$. When $\tau \in S_{n}$ and $\pi=\left(\pi_{1}, \ldots, \pi_{m}\right) \in \mathcal{B}_{n}$, we let $\tau \circ \pi:=\left(\tau\left(\pi_{1}\right), \ldots, \tau\left(\pi_{m}\right)\right)$. If $\mu$ is a signed measure on $\left(\{0,1\}^{n}, \sigma\left(\{0,1\}^{n}\right)\right)$ which is such that $\mu(\tau \circ \pi)=\mu(\pi)$ for all $\tau \in S_{n}$ and $\pi \in \mathcal{B}_{n}$, we say that $\mu$ is permutation invariant.

Finally, recall when $G=(V, E)$ is a finite graph and $w \in\{0,1\}^{E}$, we define $E_{w}:=\left\{e \in E: w_{e}=1\right\}$ and note that this defines a partition $\pi[w] \in \mathcal{B}_{V}$ if we let $v, v^{\prime} \in V$ be in the same partition element of $\pi[w]$ if and only if they are in the same connected component of the graph $\left(V, E_{w}\right)$. If $T \subseteq V$ and $|T| \geq 2$, then we let $\pi[T]$ be the unique partition in $\in \mathcal{B}_{V}$ in which $T$ is a partition element and all other partition elements are singletons. 


\subsection{The Associated Linear Operator}

Let $n \in \mathbb{N}, v \in \mathcal{P}\left(\{0,1\}^{n}\right)$ and $p=v\left(1^{\{1\}}\right)$. It was observed in [12] that if $\mu \in \operatorname{RER}_{[n]}$ is such that $\Phi_{p}(\mu)=v$, then $\mu$ and $v$ satisfy the following set of linear equations.

$$
\nu(\sigma)=\sum_{\pi \in \mathcal{B}_{n}: \pi \triangleleft \sigma} p^{\|\sigma\|_{\pi}}(1-p)^{\|\pi\|-\|\sigma\|_{\pi}} \mu(\pi), \quad \sigma \in\{0,1\}^{n} .
$$

Moreover, whenever a nonnegative measure $\mu$ on $\left(\mathcal{B}_{n}, \sigma\left(\mathcal{B}_{n}\right)\right)$ satisfies these equations, then $\mu \in \operatorname{RER}_{[n]}$ and $\Phi_{p}(\mu)=v$, i.e., then $\mu$ is a color representation of $v$. A signed measure $\mu$ on $\left(\mathcal{B}_{n}, \sigma\left(\mathcal{B}_{n}\right)\right)$ which satisfies (2), but which is not necessarily nonnegative, will be called a formal solution to (2). If we for a finite set $S$ let RER* denote the set of signed measures on $\left(\mathcal{B}_{S}, \sigma\left(B_{S}\right)\right)$ and $\mathcal{P}^{*}(\{0,1\})$ denote the set of signed measures on $\left(\{0,1\}^{n}, \sigma\left(\{0,1\}^{n}\right)\right)$, then for each $p \in(0,1)$ we can use (2) to extend $\Phi_{p}: \operatorname{RER}_{S} \rightarrow \mathcal{P}\left(\{0,1\}^{S}\right)$ to a mapping $\Phi_{S}^{*}: \operatorname{RER}_{S}^{*} \rightarrow \mathcal{P}^{*}(\{0,1\})$, whose restriction to $\operatorname{RER}_{S}$ is equal to $\Phi$.

The matrix corresponding to the system of linear equations given in (2) is given by

$$
A_{n, p}(\sigma, \pi):=\left\{\begin{array}{ll}
p^{\|\sigma\|_{\pi}}(1-p)^{\|\pi\|-\|\sigma\|_{\pi}} & \text { if } \pi \triangleleft \sigma \\
0 & \text { else }
\end{array}, \quad \sigma \in\{0,1\}^{n}, \pi \in \mathcal{B}_{n} .\right.
$$

It was shown in [8] that $A_{n, 1 / 2}$ has rank $2^{n-1}$, and that when $p \in(0,1) \backslash\{1 / 2\}$, then $A_{n, p}$ has rank $2^{n}-n$. When we use the matrix $A_{n, p}$ to think about (2) as a system of linear equations, we will abuse notation slightly and let $\mu \in \operatorname{RER}_{[n]}^{*}$ denote both the signed measure and the corresponding vector $(\mu(\pi))_{\pi \in \mathcal{B}_{n}}$, given some unspecified and arbitrary ordering of $\mathcal{B}_{n}$.

\subsection{Subsequential Limits of Color Representations}

Assume that $n \in \mathbb{N}$ and that a family $\mathcal{N}=\left(v_{p}\right)_{p \in(0,1)}$ of probability measures on $\mathcal{P}\left(\{0,1\}^{n}\right)$ are given. Further, assume that for each $p \in(0,1)$, the marginal distribution of $v_{p}$ is given by $(1-p) \delta_{0}+p \delta_{1}$. We say that a measure $\mu \in R E R_{[n]}$ arise as a subsequential limit of color representations of measures in $\mathcal{N}$ as $p \rightarrow 1 / 2$, if there is a sequence $p_{1}, p_{2}, \ldots$ in $(0,1) \backslash\{1 / 2\}$ with $\lim _{j \rightarrow \infty} p_{j}=1 / 2$ and a measure $\mu_{j} \in \Phi_{p_{j}}^{-1}\left(v_{j}\right)$ such that for all $\pi \in \mathcal{B}_{n}$ we have $\lim _{j \rightarrow \infty} \mu_{j}(\pi)=\mu(\pi)$.

\section{Color Representations of $X^{G, \beta, 0}$}

In this section, we give a proof of Theorem 1.2.

Proof of Theorem 1.2 When $p=1 / 2, \sigma \in\{0,1\}^{n}$ and $\pi \in \mathcal{B}_{n}$, then

$$
A(\sigma, \pi):=A_{n, p}(\sigma, \pi)= \begin{cases}2^{-\|\pi\|} & \text { if } \pi \triangleleft \sigma, \\ 0 & \text { otherwise. }\end{cases}
$$


For $S \subseteq[n]$ and $\pi \in \mathcal{B}_{n}$, define

$$
A^{\prime}(S, \pi):=\sum_{\sigma \in\{0,1\}^{n}:\left.\sigma\right|_{S} \equiv 1} A(\sigma, \pi)=2^{-\left\|\left.\pi\right|_{S}\right\|} .
$$

Since for any $S \subseteq[n]$ and $\pi \in \mathcal{B}_{n}$ we have

$$
\begin{aligned}
& \sum_{T \subseteq[n]: S \subseteq T} A^{\prime}(T, \pi)(-1)^{|T|-|S|}=\sum_{\substack{T \subseteq[n]: \\
S \subseteq T}} \sum_{\substack{\sigma \in\{0,1\}^{n}: \\
\left.\sigma\right|_{T} \equiv 1}} A(\sigma, \pi)(-1)^{|T|-|S|} \\
& =\sum_{\substack{\sigma \in\{0,1\}^{n}: \\
\left.\sigma\right|_{S} \equiv 1}} A(\sigma, \pi) \sum_{\substack{T \subseteq[n]: \\
\left.\sigma\right|_{T} \equiv 1}}(-1)^{|T|-|S|}=A\left(1^{S} 0^{[n] \backslash S}, \pi\right)
\end{aligned}
$$

it follows that $A$ and $A^{\prime}$ are row equivalent. Moreover, by Möbius inversion theorem, applied to the set of subsets of $[n]$ ordered by inclusion, the matrix

$$
A^{\prime \prime}(S, \pi):=\sum_{S^{\prime}: S^{\prime} \subseteq S} 2^{\left|S^{\prime}\right|}(-1)^{|S|-\left|S^{\prime}\right|} A^{\prime}\left(S^{\prime}, \pi\right), \quad S \subseteq[n], \pi \in \mathcal{B}_{n}
$$

is row equivalent to $A^{\prime}$, and hence also to $A$. By Theorem 1.2 in [8], $A$ has rank $2^{n-1}$, and hence the same is true for $A^{\prime \prime}$.

Now note that if $S \subseteq[n], \pi \in \mathcal{B}_{n}$, and we let $T_{1}, T_{2}, \ldots, T_{\left\|\left.\pi\right|_{S}\right\|}$ denote the partition elements of $\left.\pi\right|_{S}$, then

$$
\begin{aligned}
\sum_{S^{\prime}: S^{\prime} \subseteq S} 2^{\left|S^{\prime}\right|}(-1)^{|S|-\left|S^{\prime}\right|} A^{\prime}\left(S^{\prime}, \pi\right) & =2^{|S|} \sum_{S^{\prime}: S^{\prime} \subseteq S}(-2)^{\left|S^{\prime}\right|-|S|} A^{\prime}\left(S^{\prime}, \pi\right) \\
& =2^{|S|} \sum_{S^{\prime}: S^{\prime} \subseteq S}\left(-2^{-1}\right)^{|S|-\left|S^{\prime}\right|} \cdot 2^{-\| \pi \mid} S^{\prime} \| \\
& =2^{|S|} \sum_{S_{1}, \ldots, S_{m}:} \prod_{i=1}^{m}\left(-2^{-1}\right)^{\left|T_{i}\right|-\left|S_{i}\right|} 2^{-\mathbb{1}_{S_{i} \neq \emptyset}} \\
& =2^{|S|} \prod_{i=1}^{m} \sum_{S_{i}: S_{i} \subseteq T_{i}}\left(-2^{-1}\right)^{\left|T_{i}\right|-\left|S_{i}\right|} \cdot 2^{-\mathbb{1}_{S_{i} \neq \emptyset}} \\
& =2^{|S|} \prod_{i=1}^{m}\left(1+(-1)^{\left|T_{i}\right|}\right) \cdot\left(2^{-1}\right)^{\left|T_{i}\right|+1} \\
& =\mathbb{1}\left(\left.\pi\right|_{S} \text { has only even-sized partition elements }\right) .
\end{aligned}
$$

and hence

$$
A^{\prime \prime}(S, \pi)=\mathbb{1}\left(\left.\pi\right|_{S} \text { has only even-sized partition elements }\right) .
$$


Let $T$ be a spanning tree of $G$. Let $\mathcal{B}_{n}^{T} \subseteq \mathcal{B}_{n}$ denote the partitions of $[n]$ whose partition elements induce connected subgraphs of $T$. Note that the number of such partitions is equal to $2^{n-1}$. For $S \subseteq[n]$ with $|S|$ even and $\pi \in \mathcal{B}_{n}^{T}$, define

$$
A_{T}(S, \pi):=\mathbb{1}\left(\left.\pi\right|_{S}\right. \text { has only even-sized partition elements). }
$$

Then, $A_{T}$ is a submatrix of $A^{\prime \prime}$. We will show that $A_{T}$ has full rank. Since $A_{T}$ is a $2^{n-1}$ by $2^{n-1}$ matrix, this is equivalent to having nonzero determinant. To see that $\operatorname{det} A_{T} \neq 0$, note first that if $S \subseteq[n],|S|$ is even and $\pi \in \mathcal{B}_{n}^{T}$, then

$$
\begin{aligned}
B(S, \pi) & :=\sum_{\pi^{\prime}: \pi^{\prime} \triangleleft \pi}(-1)^{|\pi|-\left|\pi^{\prime}\right|} A_{T}\left(S, \pi^{\prime}\right) \\
& =\mathbb{1}\left(\begin{array}{c}
\pi \text { has only even-sized partition elements } \\
\text { and finer partition of } S \text { has at least } \\
\text { one odd-sized partition element }
\end{array}\right) .
\end{aligned}
$$

Since all partition elements of $\pi \in \mathcal{B}_{n}^{T}$ induce connected subgraphs of $G, B$ is a permutation matrix. Since all permutation matrices have nonzero determinant, this implies that $B$, and hence also $A_{T}$, has full rank.

Since $A_{T}$ has $2^{n-1}$ rows and columns, this implies, in particular, that $A_{T}$ has rank $2^{n-1}$. On the other hand, $A_{T}$ is a submatrix of $A^{\prime \prime}$, and $A^{\prime \prime}$ is row equivalent to $A$ which also has rank $2^{n-1}$. This implies, in particular, that when we solve (2), we can use the columns corresponding to partitions in $\mathcal{B}_{n}^{T}$ as dependent variables.

Now recall that since $X^{G, \beta, 0}$ is the Ising model on some graph $G, X^{G, \beta, 0}$ has at least one color representation given by $\mu_{G, 1-e^{-2 \beta}}$. The random cluster model $\mu_{G, 1-e^{-2 \beta}}$ gives strictly positive mass to all partitions $\pi \in \mathcal{B}_{n}$ whose partition elements induce connected subgraphs of $G$. In particular, it gives strictly positive mass to all partitions in $\mathcal{B}_{n}^{T}$. If we use the columns corresponding to partitions in $\mathcal{B}_{n}^{T}$ as dependent variables, then all dependent variables are given positive mass by $\mu_{G, 1-e^{-2 \beta}}$. Since $n \geq 3$, there is at least one free variable. By continuity, it follows that we can find another color representation by increasing the value of this free variable a little. If $G$ is not a tree, there will be at least of free variable corresponding to a partition $\pi \in \mathcal{B}_{n} \backslash \mathcal{B}^{T}$, whose partition elements induce connected subgraphs of $G$ (but not $T$ ). From this the desired conclusion follows.

Remark 3.1 It is not the case that all sets of $2^{n-1}$ columns of $A_{n, 1 / 2}$ have full rank. To see this, note first that there are exactly $\left|\mathcal{B}_{n}\right|-\left|\mathcal{B}_{n-1}\right|$ partitions in $\mathcal{B}_{n}$ in which 1 is not a singleton. If $\left|\mathcal{B}_{n}\right|-\left|\mathcal{B}_{n-1}\right| \geq 2^{n-1}$, then there is a set $\mathcal{B}^{\prime} \subseteq \mathcal{B}_{n}$ of such partitions of size $2^{n-1}$. An easy calculation shows that this happens whenever $n \geq 4$. Let $\mu \in \operatorname{RER}_{[n]}^{*}$ be a signed measure on $\mathcal{B}_{n}$ with support only on $\mathcal{B}^{\prime}$, and let $v:=\Phi_{1 / 2}^{*}(\mu)$. Then, by definition, if $v \in \mathcal{P}\left(\{0,1\}^{n}\right)$ then if $v\left(1^{\{1\}} 0^{[n] \backslash\{1\}}\right)=0$. In particular, this implies that the columns of $A_{n, 1 / 2}$ corresponding to the partitions in $\mathcal{B}^{\prime}$ cannot have full rank. 


\section{Color Representations of $X^{K_{n}, \beta, h}$ for $n \in\{3,4,5\}$}

In this section, we provide proofs of Propositions 1.3 and 1.4. In both cases, Mathematica was used to simplify the formulas for the color representations for different values of $\beta$ and $h$.

Proof of Proposition 1.3 Fix $h>0$ and let $p=v_{K_{3}, \beta, h}\left(1^{\{1\}}\right)$. Note that since $\left|V\left(K_{3}\right)\right|=3$, the relevant set of partitions is given by

$$
\mathcal{B}_{3}=\{(\{1,2,3\}),(\{1,2\},\{3\}),(\{1\},\{2,3\}),(\{1,3\},\{2\}),(\{1\},\{2\},\{3\})\} .
$$

By Theorem 1.4 in [8], the linear equation system $A_{3, p} \mu=v_{K_{3}, \beta, h}$ has a unique formal solution $\mu \in R E R_{[3]}^{*}$. With some work, one verifies that this solution satisfies

$$
\mu((\{1,2\},\{3\}))=\mu((\{1,3\},\{2\}))=\mu((\{1\},\{2,3\})),
$$

and

$$
\begin{aligned}
& \mu((\{1,2,3\})) \\
& =\frac{\left(e^{4 \beta}-1\right)^{2} e^{4 h}\left(e^{4 \beta}+e^{4 \beta+2 h}+e^{4 \beta+4 h}+5 e^{2 h}+2 e^{4 h}+2\right)}{\left(e^{4 \beta}+2 e^{2 h}+e^{4 h}\right)\left(e^{4 \beta+4 h}+2 e^{2 h}+1\right)\left(e^{4 \beta}+e^{4 \beta+2 h}+e^{4 \beta+4 h}+e^{2 h}\right)} \\
& \mu((\{1\},\{2,3\})) \\
& =\frac{\left(e^{4 \beta}-1\right) e^{2 h}\left(e^{2 h}+1\right)^{2}\left(e^{4 \beta}-e^{4 \beta+2 h}+e^{4 \beta+4 h}+3 e^{2 h}\right)}{\left(e^{4 \beta}+2 e^{2 h}+e^{4 h}\right)\left(e^{4 \beta+4 h}+2 e^{2 h}+1\right)\left(e^{4 \beta}+e^{4 \beta+2 h}+e^{4 \beta+4 h}+e^{2 h}\right)} \\
& \mu((\{1\},\{2\},\{3\}))= \\
& \frac{\left(e^{2 h}+1\right)^{2}\left(e^{4 \beta}-e^{4 \beta+2 h}+e^{4 \beta+4 h}+3 e^{2 h}\right)^{2}}{\left(e^{4 \beta}+2 e^{2 h}+e^{4 h}\right)\left(e^{4 \beta+4 h}+2 e^{2 h}+1\right)\left(e^{4 \beta}+e^{4 \beta+2 h}+e^{4 \beta+4 h}+e^{2 h}\right)} .
\end{aligned}
$$

Since $e^{4 \beta+4 h} \geq e^{4 \beta+2 h}$, it is immediately clear that for all $\pi \in \mathcal{B}_{3}, \beta>0$ and $h>0$, $\mu(\pi)$ is nonnegative, and hence (i) holds.

By letting $h \rightarrow 0$ in the expression for $\mu((\{1\},\{2\},\{3\}))$, while keeping $\beta$ fixed, one obtains

$$
\lim _{h \rightarrow 0} \mu((\{1\},\{2\},\{3\}))=\frac{4}{1+3 e^{4 \beta}} .
$$

On the other hand, it is easy to check that

$$
\mu_{K_{3}, e^{-2 \beta}}((\{1\},\{2\},\{3\}))=\frac{4 e^{-2 \beta}}{3+e^{4 \beta}} .
$$

Since these two expressions are not equal for any $\beta>0$, (ii) holds. 
Proof of Proposition 1.4 Fix $h>0$ and let $p:=v_{K_{4}, \beta, h}\left(1^{\{1\}}\right)$. Since $v_{K_{4}, \beta, h}$ is permutation invariant, it follows that if $v_{K_{4}, \beta, h}$ has a color representation, then it has at least one color representation which is invariant under the action of $S_{4}$. It is easy to check that there are exactly five different partitions in $\mathcal{B}_{4} / S_{4}$, namely $(\{1,2,3,4\}),(\{1,2,3\},\{4\}),(\{1,2\},\{3,4\}),(\{1\},\{2\},\{3,4\})$ and $(\{1\},\{2\},\{3\},\{4\})$. By Theorem 1.5(i) in [8], the linear subspace spanned by all $S_{4}$-invariant formal solutions $\mu$ of $A_{4, p} \mu=v_{K_{4}, \beta, h}$ has dimension one. By linearity, this implies, in particular, that if $v_{K_{4}, \beta, h}$ has a color representation, then it has at least one color representation which is $S_{4}$-invariant and gives zero mass to at least one of the partitions in $\mathcal{B}_{4} / S_{4}$. This gives us one unique solution for each choice of partition in $\mathcal{B}_{4} / S_{4}$.

Solving the corresponding linear equation systems in Mathematica and studying the solutions, after some work, one obtains the desired necessary and sufficient condition.

Remark 4.1 With the same strategy as in the proof of Proposition 1.4, one can find analogous necessary and sufficient conditions for $G=K_{n}$ when $n \geq 5$. However, already when $n=5$ the analogue inequality is quite complicated; in this case, one can show that a necessary and sufficient condition to have a color representation is that

$$
\begin{aligned}
& -x^{18} y^{10}-x^{18} y^{8}+x^{18} y^{7}-x^{18} y^{6}-x^{18} y^{4}+x^{16} y^{14}+x^{16} y^{12}+x^{16} y^{9} \\
& +3 x^{16} y^{8}+2 x^{16} y^{7}+3 x^{16} y^{6}+x^{16} y^{5}+x^{16} y^{2}+x^{16}-3 x^{14} y^{11} \\
& -x^{14} y^{10}-12 x^{14} y^{9}+6 x^{14} y^{8}-11 x^{14} y^{7}+6 x^{14} y^{6}-12 x^{14} y^{5}-x^{14} y^{4} \\
& -3 x^{14} y^{3}+9 x^{12} y^{13}-3 x^{12} y^{12}+6 x^{12} y^{11}+7 x^{12} y^{10}+15 x^{12} y^{9}-4 x^{12} y^{8} \\
& +18 x^{12} y^{7}-4 x^{12} y^{6}+15 x^{12} y^{5}+7 x^{12} y^{4}+6 x^{12} y^{3}-3 x^{12} y^{2}+9 x^{12} y \\
& +19 x^{10} y^{12}+27 x^{10} y^{11}+14 x^{10} y^{10}+36 x^{10} y^{9}-21 x^{10} y^{8}+45 x^{10} y^{7}-21 x^{10} y^{6} \\
& +36 x^{10} y^{5}+14 x^{10} y^{4}+27 x^{10} y^{3}+19 x^{10} y^{2}+20 x^{8} y^{12}-18 x^{8} y^{11}+15 x^{8} y^{10} \\
& -11 x^{8} y^{9}+50 x^{8} y^{8}-102 x^{8} y^{7}+50 x^{8} y^{6}-11 x^{8} y^{5}+15 x^{8} y^{4}-18 x^{8} y^{3} \\
& +20 x^{8} y^{2}+82 x^{6} y^{11}+83 x^{6} y^{10}+76 x^{6} y^{9}+178 x^{6} y^{8}+197 x^{6} y^{7}+178 x^{6} y^{6} \\
& +76 x^{6} y^{5}+83 x^{6} y^{4}+82 x^{6} y^{3}+54 x^{4} y^{10}+226 x^{4} y^{9}+152 x^{4} y^{8}+386 x^{4} y^{7} \\
& +152 x^{4} y^{6}+226 x^{4} y^{5}+54 x^{4} y^{4}-84 x^{2} y^{9}+12 x^{2} y^{8}-156 x^{2} y^{7}+12 x^{2} y^{6} \\
& -84 x^{2} y^{5}-72 y^{8}-56 y^{7}-72 y^{6} \geq 0
\end{aligned}
$$

where $x=e^{2 \beta}$ and $y=e^{2 h}$. 


\section{Color Representations for small $h>0$}

\subsection{Existence}

The goal of this subsection is to provide a proof of Theorem 1.6. A main tool in the proof of this theorem will be the following result from [8].

Theorem 5.1 (Theorem 1.7 in [8]) Let $n \in \mathbb{N}$ and let $\left(v_{p}\right)_{p \in(0,1)}$ be a family of probability measures on $\{0,1\}^{n}$. For each $p \in(0,1)$, assume that $v_{p}$ has marginals $p \delta_{1}+(1-p) \delta_{0}$, and that for each $S \subseteq[n], v_{p}\left(1^{S}\right)$ is two times differentiable in $p$ at $p=1 / 2$. Assume further that for any $S \subseteq T \subseteq[n]$ and any $p \in(0,1)$, we have that

$$
v_{p}\left(0^{S} 1^{T \backslash S}\right)=v_{1-p}\left(0^{T \backslash S} 1^{S}\right) .
$$

Then, the set of solutions $\left\{(\mu(\pi))_{\pi \in \mathcal{B}_{n}}\right\}$ to (4) which arise as subsequential limits of solutions to (2) as $p \rightarrow 1 / 2$ is exactly the set of solutions to the system of equations

$$
\begin{cases}\sum_{\pi \in \mathcal{B}_{n}} 2^{-\|\pi\|} \mu(\pi)=v_{1 / 2}\left(1^{S}\right), & S \subseteq[n],|S| \text { even } \\ \sum_{\pi \in \mathcal{B}_{n}}\|\pi\|_{S} 2^{-\left\|\left.\pi\right|_{S}\right\|+1} \mu(\pi)=v_{1 / 2}^{\prime}\left(1^{S}\right), & S \subseteq[n],|S| \text { odd }\end{cases}
$$

Remark 5.2 By applying elementary row operations to the system of linear equations in (9), one obtains the following equivalent system of linear equations.

$$
\begin{aligned}
& \sum_{\pi \in \mathcal{B}_{n}} \mathbb{1}\left(\left.\pi\right|_{S} \text { has at most one odd-sized partition element }\right) \mu(\pi) \\
& \quad= \begin{cases}\sum_{S^{\prime} \subseteq S}(-2)^{\left|S^{\prime}\right|+1} v_{1 / 2}^{\prime}\left(1^{S^{\prime}}\right) & \text { if }|S| \text { is odd } \\
\sum_{S^{\prime} \subseteq S}(-2)^{\left|S^{\prime}\right|} v_{1 / 2}\left(1^{S^{\prime}}\right) & \text { if }|S| \text { is even. }\end{cases}
\end{aligned}
$$

(See the last equation in the proof of Theorem 1.7 in [8].)

The proof of Theorem 1.6 will be divided into two parts, corresponding to the two cases $\hat{\beta}>1$ and $\hat{\beta}=1$.

Proof of Theorem 1.6 when $\hat{\beta}>1$ (the supercritical regime). Let $z_{\hat{\beta}}$ be the unique positive root to the equation $z=\tanh (\hat{\beta} z)$. Then, it is well known (see, e.g., [7], p. 181) that as $n \rightarrow \infty$, we have

$$
\frac{2\left\|X^{K_{n}, \beta, 0}\right\|-n}{n} \stackrel{d}{\rightarrow} \frac{1}{2} \delta_{-z_{\hat{\beta}}}+\frac{1}{2} \delta_{z_{\hat{\beta}}}
$$


and hence, by Lemma 7.4, as $n \rightarrow \infty$ we have that

$$
\left\{\begin{array}{l}
\sum_{\sigma \in\{0,1\}^{n}} \chi_{\emptyset}(\sigma) v_{K_{n}, \beta, 0}(\sigma)=1 \\
\sum_{\sigma \in\{0,1\}^{n}}(2\|\sigma\|-n) \chi_{\{1\}}(\sigma) v_{K_{n}, \beta, 0}(\sigma) \sim n z_{\hat{\beta}}^{2} \\
\sum_{\sigma \in\{0,1\}^{n}} \chi_{[2]}(\sigma) v_{K_{n}, \beta, 0}(\sigma) \sim z_{\hat{\beta}}^{2} \\
\sum_{\sigma \in\{0,1\}^{n}}(2\|\sigma\|-n) \chi_{[3]}(\sigma) v_{K_{n}, \beta, 0}(\sigma) \sim n z_{\hat{\beta}}^{4} \\
\sum_{\sigma \in\{0,1\}^{n}} \chi_{[4]}(\sigma) v_{K_{n}, \beta, 0}(\sigma) \sim z_{\hat{\beta}}^{4} .
\end{array}\right.
$$

By Remark 5.2, any color representation $\mu$ of $v_{K_{n}, \beta, 0}$ which arise as a subsequential limit of color representations of $v_{K_{n}, \beta, h}$ as $h \rightarrow 0$ must satisfy (10). By Lemma 7.3, for any $S \subseteq[n]$ we have that

$$
\sum_{S^{\prime} \subseteq S}(-2)^{|S|} v_{K_{n}, \beta, 0}\left(1^{S^{\prime}}\right)=(-1)^{|S|} \sum_{\sigma \in\{0,1\}^{n}} \chi_{S}(\sigma) v_{K_{n}, \beta, 0}(\sigma)
$$

and

$$
\sum_{S^{\prime} \subseteq S}(-2)^{|S|+1} v_{K_{n}, \beta, 0}^{\prime}\left(1^{S^{\prime}}\right)=(-1)^{|S|+1} \frac{\sum_{\sigma \in\{0,1\}^{n}}(2\|\sigma\|-n) \chi_{S}(\sigma) v_{K_{n}, \beta, 0}(\sigma)}{\sum_{\sigma \in\{0,1\}^{n}}(2\|\sigma\|-n) \chi_{\{1\}}(\sigma) v_{K_{n}, \beta, 0}(\sigma)}
$$

and hence (10) is equivalent to that

$$
\begin{gathered}
\sum_{\pi \in \mathcal{B}_{n}} \mathbb{1}\left(\left.\pi\right|_{S} \text { has at most one odd-sized partition element }\right) \mu(\pi) \\
=\lambda_{S}^{(n)}:= \begin{cases}\frac{\sum_{\sigma \in\{0,1\}^{n}}(2\|\sigma\|-n) \chi_{S}(\sigma) v_{K_{n}, \beta, 0}(\sigma)}{\sum_{\sigma \in\{0,1\}^{n}}(2\|\sigma\|-n) \chi_{\{1\}}(\sigma) v_{K_{n}, \beta, 0}(\sigma)} & \text { if }|S| \text { is odd } \\
\sum_{\sigma \in\{0,1\}^{n}} \chi_{S}(\sigma) v_{K_{n}, \beta, 0}(\sigma) & \text { if }|S| \text { is even. }\end{cases}
\end{gathered}
$$

Note that since $v_{K_{n}, \beta, 0}$ is permutation invariant, we have $\lambda_{S}^{(n)}=\lambda_{[|S|]}^{(n)}$ for any $S \subseteq[n]$. This implies, in particular, that if (15) has a nonnegative solution, then it must have at least one permutation invariant nonnegative solution $\mu^{(n)}$. Observe that $\left.\mu^{(n)}\right|_{\mathcal{B}_{4}}$ satisfies (15) for any set $S \subseteq\{1,2,3,4\}$. For this reason, for each $n \geq 4$, we now consider the system of linear equations given by

$$
\begin{aligned}
& \left\{\begin{array}{l}
\sum_{\pi \in \mathcal{B}_{4}} \mathbb{1}\left(\left.\pi\right|_{S} \text { has at most one odd-sized partition element }\right) \mu(\pi)=\lambda_{S}^{(n)} \\
\mu(\pi)=\mu(\tau \circ \pi) \text { for all } \tau \in S_{n}
\end{array}\right. \\
& \quad S \subseteq[4]
\end{aligned}
$$

Let $A^{(4)}$ denote the corresponding matrix. By Theorem 1.5(i) in [8], the null space of $A^{(4)}$ has dimension one. Since (13) is a set of linear equations, this implies that if a nonnegative solution exists, then there will exist a nonnegative solution $\mu$ in which either $\mu((\{1,2,3,4\})), \mu((\{1,2,3\},\{4\})), \mu((\{1,2\},\{3,4\})), \mu((\{1,2\},\{3\},\{4\}))$ or 
$\mu((\{1\},\{2\},\{3\},\{4\}))$ is equal to zero. Next, note that when $S \subseteq[n]$ satisfies $|S| \leq 4$, by (11), we have that $\lambda_{S}^{(\infty)}:=\lim _{n \rightarrow \infty} \lambda_{S}^{(n)}=z_{\hat{\beta}}^{2\lfloor|S| / 2\rfloor}$. Define $\lambda^{(n)}=\left(\lambda_{S}^{(n)}\right)_{S \subseteq[4]}$ and $\lambda^{(\infty)}=\left(\lambda_{S}^{(\infty)}\right)_{S \subseteq[4]}$. Using these definitions, one verifies that the five (permutation invariant) solutions $\mu_{1}^{(\infty)}, \mu_{2}^{(\infty)}, \mu_{3}^{(\infty)}, \mu_{4}^{(\infty)}$ and $\mu_{5}^{(\infty)}$ to

$$
A^{(4)} \mu=\lambda^{(\infty)}
$$

with at least one zero entry are given by

$$
\begin{aligned}
& \mu_{1}^{(\infty)}((\{1,2,3,4\}),(\{1\},\{2,3,4\}),(\{1,2\},\{3,4\}),(\{1\},\{2\},\{3,4\}),(\{1\},\{2\},\{3\},\{4\})) \\
& \quad=\left(0, z_{\hat{\beta}}^{2}, z_{\hat{\beta}}^{4} / 3,-z_{\hat{\beta}}^{2}\left(1+z_{\hat{\beta}}^{2} / 3\right),\left(1+z_{\hat{\beta}}^{2}\right)^{2}\right) \\
& \mu_{2}^{(\infty)}((\{1,2,3,4\}),(\{1\},\{2,3,4\}),(\{1,2\},\{3,4\}),(\{1\},\{2\},\{3,4\}),(\{1\},\{2\},\{3\},\{4\})) \\
& \quad=\left(z_{\hat{\beta}}^{2}, 0, z_{\hat{\beta}}^{2}\left(z_{\hat{\beta}}^{2}-1\right) / 3,-z_{\hat{\beta}}^{2}\left(z_{\hat{\beta}}^{2}-1\right) / 3,\left(z_{\hat{\beta}}^{2}-1\right)^{2}\right) \\
& \mu_{3}^{(\infty)}((\{1,2,3,4\}),(\{1\},\{2,3,4\}),(\{1,2\},\{3,4\}),(\{1\},\{2\},\{3,4\}),(\{1\},\{2\},\{3\},\{4\})) \\
& \quad=\left(z_{\hat{\beta}}^{4},-z_{\hat{\beta}}^{2}\left(z_{\hat{\beta}}^{2}-1\right), 0, z_{\hat{\beta}}^{2}\left(z_{\hat{\beta}}^{2}-1\right),-\left(z_{\hat{\beta}}^{2}-1\right)\left(1+3 z_{\hat{\beta}}^{2}\right)\right) \\
& \mu_{4}^{(\infty)}((\{1,2,3,4\}),(\{1\},\{2,3,4\}),(\{1,2\},\{3,4\}),(\{1\},\{2\},\{3,4\}),(\{1\},\{2\},\{3\},\{4\})) \\
& \quad=\left(z_{\hat{\beta}}^{2}\left(3+z_{\hat{\beta}}^{2}\right) / 4,-z_{\hat{\beta}}^{2}\left(z_{\hat{\beta}}^{2}-1\right) / 4, z_{\hat{\beta}}^{2}\left(z_{\hat{\beta}}^{2}-1\right) / 4,0,-\left(z_{\hat{\beta}}^{2}-1\right)\right)
\end{aligned}
$$

and

$$
\begin{aligned}
& \mu_{5}^{(\infty)}((\{1,2,3,4\}),(\{1\},\{2,3,4\}),(\{1,2\},\{3,4\}),(\{1\},\{2\},\{3,4\}), \\
& \quad(\{1\},\{2\},\{3\},\{4\})) \\
& \quad=\left(\left(1+z_{\hat{\beta}}^{2}\right)^{2} / 4,-\left(z_{\hat{\beta}}^{2}-1\right)^{2} / 4,\left(z_{\hat{\beta}}^{2}-1\right)\left(1+3 z_{\hat{\beta}}^{2}\right) / 12,-\left(z_{\hat{\beta}}^{2}-1\right) / 3,0\right) .
\end{aligned}
$$

Above, all the negative entries are displayed in red. Since all of these solutions have at least one strictly negative entry, it follows that any solutions $\mu^{(\infty)}$ to $A^{(4)} \mu^{(\infty)}=\lambda^{(\infty)}$ must have at least one strictly negative entry.

Now let $\mu^{(n)}$ be a permutation invariant solution to (15). Then, $\left.\mu^{(n)}\right|_{\mathcal{B}_{4}}$ must satisfy $\left.A^{(4)} \mu^{(n)}\right|_{\mathcal{B}_{4}}=\lambda^{(n)}$. But this implies that for all $S \subseteq[4]$ we have that

$$
\lim _{n \rightarrow \infty}\left(\left.A^{(4)} \mu^{(n)}\right|_{\mathcal{B}_{4}}-A^{(4)} \mu^{(\infty)}\right)(S)=\lim _{n \rightarrow \infty} \lambda^{(n)}(S)-\lambda^{(\infty)}(S)=0 .
$$

Since

$$
\lim _{n \rightarrow \infty}\left(\left.A^{(4)} \mu^{(n)}\right|_{\mathcal{B}_{4}}-A^{(4)} \mu^{(\infty)}\right)(S)=A^{(4)}\left(\left.\lim _{n \rightarrow \infty} \mu^{(n)}\right|_{\mathcal{B}_{4}}-\mu^{(\infty)}\right)(S)
$$

this show that

$$
\left.\lim _{n \rightarrow \infty} \mu^{(n)}\right|_{\mathcal{B}_{4}}-\mu^{(\infty)} \in \operatorname{Null} A^{(4)}
$$


This, in particular, implies that $\mu^{(n)}$ must have negative entries for all sufficiently large $n$, and hence, the desired conclusion follows.

We now give a proof of Theorem 1.6 in the case $\hat{\beta}=1$. This proof is very similar to the previous proof, but requires that we look at the distribution of the first five coordinates of $X_{K_{n}, \beta, 0}$ and are more careful with the asymptotics.

Proof of Theorem 1.6 when $\hat{\beta}=1$ (the critical regime). Assume that $n$ is large. By Theorem V.9.5 in [7], as $n \rightarrow \infty$, we have that

$$
\frac{2\left\|X^{K_{n}, \beta, 0}\right\|-n}{n^{3 / 4}} \stackrel{d}{\rightarrow} X
$$

where $X$ is a random variable with probability density function $f(x)=\frac{3^{1 / 4} \Gamma(1 / 4)}{\sqrt{2}}$ $e^{-x^{4} / 12}, x \in \mathbb{R}$.

Let $m_{1}^{(n)}, m_{2}^{(n)}$, etc., be the moments of $X^{K_{n}, \beta, 0}$. By Lemma 7.4, as $n \rightarrow \infty$, we have that

$$
\left\{\begin{array}{l}
\sum_{\sigma \in\{0,1\}^{n}} \chi_{\emptyset}(\sigma) v_{K_{n}, \beta, 0}(\sigma)=1 \\
\sum_{\sigma \in\{0,1\}^{n}}(2\|\sigma\|-n) \chi_{\{1\}}(\sigma) v_{K_{n}, \beta, 0}(\sigma) \sim n^{1 / 2} m_{2}^{(n)} \\
\sum_{\sigma \in\{0,1\}^{n}} \chi_{[2]}(\sigma) v_{K_{n}, \beta, 0}(\sigma) \sim n^{-1 / 2} m_{2}^{(n)} \\
\sum_{\sigma \in\{0,1\}^{n}}(2\|\sigma\|-n) \chi_{[3]}(\sigma) v_{K_{n}, \beta, 0}(\sigma) \sim m_{4}^{(n)} \\
\sum_{\sigma \in\{0,1\}^{n}} \chi_{[4]}(\sigma) v_{K_{n}, \beta, 0}(\sigma) \sim n^{-1} m_{4}^{(n)} \\
\sum_{\sigma \in\{0,1\}^{n}}(2\|\sigma\|-n) \chi_{[5]}(\sigma) v_{K_{n}, \beta, 0}(\sigma) \sim n^{-1 / 2} m_{6}^{(n)} .
\end{array}\right.
$$

For each $S \subseteq[n]$, let $\lambda_{S}^{(n)}$ be defined by (15) and define $\lambda^{(n)}=\left(\lambda_{S}^{(n)}\right)_{S \subseteq[n]}$. Further, let $\hat{\lambda}^{(n)}$ be the vector containing only the largest order term of each entry of $\lambda^{(n)}$, i.e., let

$$
\left\{\begin{array}{l}
\hat{\lambda}^{(n)}(\emptyset)=1 \\
\hat{\lambda}^{(n)}([1])=1 \\
\hat{\lambda}^{(n)}([2])=n^{-1 / 2} m_{2}^{(n)} \\
\hat{\lambda}^{(n)}([3])=n^{-1 / 2} m_{4}^{(n)} / m_{2}^{(n)} \\
\hat{\lambda}^{(n)}([4])=n^{-1} m_{4}^{(n)} \\
\hat{\lambda}^{(n)}([5])=n^{-1} m_{6}^{(n)} / m_{2}^{(n)}
\end{array}\right.
$$

Then, we have that $\hat{\lambda}^{(n)}(S)-\lambda^{(n)}(S)=o\left(n^{-1 / 2}\right)$ for all $S \subseteq[n]$.

We now proceed as in the supercritical case. By Remark 5.2, any color representation $\mu$ of $v_{K_{n}, \beta, 0}$ which arise as a subsequential limit of color representations of $\nu_{K_{n}, \beta, h}$ as $h \rightarrow 0$ must satisfy (10). By Lemma 7.3, for any $S \subseteq[n]$ we have that

$$
\sum_{S^{\prime} \subseteq S}(-2)^{|S|} v_{K_{n}, \beta, 0}\left(1^{S^{\prime}}\right)=(-1)^{|S|} \sum_{\sigma \in\{0,1\}^{n}} \chi_{S}(\sigma) v_{K_{n}, \beta, 0}(\sigma)
$$


and

$$
\sum_{S^{\prime} \subseteq S}(-2)^{|S|+1} v_{K_{n}, \beta, 0}^{\prime}\left(1^{S^{\prime}}\right)=(-1)^{|S|+1} \frac{\sum_{\sigma \in\{0,1\}^{n}}(2\|\sigma\|-n) \chi_{S}(\sigma) v_{K_{n}, \beta, 0}(\sigma)}{\sum_{\sigma \in\{0,1\}^{n}}(2\|\sigma\|-n) \chi_{\{1\}}(\sigma) v_{K_{n}, \beta, 0}(\sigma)}
$$

and hence (10) is equivalent to that

$$
\begin{gathered}
\sum_{\pi \in \mathcal{B}_{n}} \mathbb{1}\left(\left.\pi\right|_{S} \text { has at most one odd-sized partition element }\right) \mu(\pi) \\
=\lambda_{S}^{(n)}:= \begin{cases}\frac{\sum_{\sigma \in\{0,1\}^{n}}(2\|\sigma\|-n) \chi_{S}(\sigma) v_{K_{n}, \beta, 0}(\sigma)}{\sum_{\sigma \in\{0,1\}^{n}}(2\|\sigma\|-n) \chi_{\{1\}}(\sigma) \nu_{K_{n}, \beta, 0}(\sigma)} & \text { if }|S| \text { is odd } \\
\sum_{\sigma \in\{0,1\}^{n}} \chi_{S}(\sigma) \nu_{K_{n}, \beta, 0}(\sigma) & \text { if }|S| \text { is even. }\end{cases}
\end{gathered}
$$

Note that $v_{K_{n}, \beta, 0}$ is permutation invariant, and hence $\lambda_{S}^{(n)}=\lambda_{[|S|]}^{(n)}$ for any $S \subseteq[n]$. This implies, in particular, that if (15) has a nonnegative solution, then it must have at least one permutation invariant nonnegative solution $\mu^{(n)}$. Observe that $\left.\mu^{(n)}\right|_{\mathcal{B}_{5}}$ satisfies (15) for any set $S \subseteq\{1,2,3,4,5\}$. For this reason, for each $n \geq 5$, we now consider the system of linear equations given by

$\left\{\begin{array}{l}\sum_{\pi \in \mathcal{B}_{5}} \mathbb{1}\left(\left.\pi\right|_{S} \text { has at most one odd-sized partition element) } \mu(\pi)=\lambda_{S}^{(n)}, \quad S \subseteq[5]\right. \\ \mu(\pi)=\mu(\tau \circ \pi) \text { for all } \tau \in S_{n}\end{array}\right.$

Let $A^{(5)}$ denote the corresponding matrix and define

$$
\begin{aligned}
\Pi_{5}:= & \{(\{1,2,3,4,5\}),(\{1,2,3,4\},\{5\}),(\{1,2,3\},\{4,5\}),(\{1,2,3\},\{4\},\{5\}), \\
& (\{1,2\},\{3,4\},\{5\}),(\{1,2\},\{3\},\{4\},\{5\}),(\{1\},\{2\},\{3\},\{4\},\{5\})\} \subseteq \mathcal{B}_{5} .
\end{aligned}
$$

By Theorem 2.2(i) in [8], the null space of $A^{(5)}$ has dimension two. This implies that if a nonnegative solution $\mu$ to (16) exists, then there will exist two district partitions $\pi^{\prime}, \pi^{\prime \prime} \in \Pi_{5}$ and a nonnegative solution $\mu$ to (16) such that at $\mu\left(\pi^{\prime}\right)=\mu\left(\pi^{\prime \prime}\right)=0$.

Let $\mu_{1}^{(n)}, \mu_{2}^{(n)}, \ldots, \mu_{\left(\begin{array}{c}7 \\ 2\end{array}\right)}^{(n)} \in R E R_{[5]}^{*}$ be the solutions of (16) which are such that at least two of $\mu((\{1,2,3,4,5\})), \mu((\{1,2,3,4\},\{5\})), \mu((\{1,2,3\},\{4,5\}))$, $\mu((\{1,2,3\},\{4\},\{5\})), \mu((\{1,2\},\{3,4\},\{5\})), \mu((\{1,2\},\{3\},\{4\},\{5\}))$ and $\mu((\{1\},\{2\},\{3\},\{4\},\{5\}))$ are equal to zero, and let $\hat{\mu}_{1}^{(n)}, \hat{\mu}_{2}^{(n)}, \ldots, \hat{\mu}_{\left(\begin{array}{c}7 \\ 2\end{array}\right)}^{(n)} \in R E R_{[5]}^{*}$ be the corresponding solutions if we replace $\lambda^{(n)}$ with $\hat{\lambda}^{(n)}$ in (16). Then, one verifies that there is an absolute constant $C>0$ such that for all $n \geq 5$ and any $i \in\left\{1,2, \ldots,\left(\begin{array}{l}7 \\ 2\end{array}\right)\right\}$ there is $\pi \in \mathcal{B}_{5}$ such that $\hat{\mu}_{i}^{(n)}(\pi)<-C n^{-1 / 2}+o\left(n^{-1 / 2}\right)$. Next, note that for any $i \in\left\{1,2, \ldots,\left(\begin{array}{l}7 \\ 2\end{array}\right)\right\}$ and $S \subseteq[5]$, we have

$$
A^{(5)} \mu_{i}^{(n)}(S)-A^{(5)} \hat{\mu}_{i}^{(n)}(S)=\lambda^{(n)}(S)-\hat{\lambda}^{(n)}(S)=o\left(n^{-1 / 2}\right),
$$


Since $A^{(5)}$ does not depend on $n$, it follows that $\mu_{i}(S)-\hat{\mu}_{i}(S)=o\left(n^{-1 / 2}\right)$ for all $S \subseteq$ [5], implying, in particular, that $\mu_{i}^{(n)}$ has the same sign as the corresponding entry of $\hat{\mu}_{i}$ at at least one entry which is negative in $\hat{\mu}_{i}$. This gives the desired conclusion.

\subsection{The Random Cluster Model is Almost Never a Limiting Color Representation}

In this section, we give a proof of Theorem 1.5. A main tool in the proof of this result will be the next lemma. The main idea of the proof of Theorem 1.5 will be to show that the necessary condition given by this lemma does in fact not hold for the random cluster model.

Throughout this section, we will use the following notation. For a fixed $n \in \mathbb{N}$, a set $S \subseteq[n]$ and a partition $\pi \in \mathcal{B}_{n}$, define

$$
A(S, \pi):=\mathbb{1}\left(\left.\pi\right|_{S} \text { has at most one odd-sized partition element }\right) .
$$

If $|S|$ is odd and $A(S, \pi)=1$, let $T_{S, \pi}$ denote the unique odd-sized partition element of $\left.\pi\right|_{S}$.

Lemma 5.3 Let $n \in \mathbb{N}$ and let $G$ be a graph with $n$ vertices. Let $\beta>0$ and assume that $\mu \in \Phi_{1 / 2}^{-1}\left(v_{G, \beta, 0}\right)$ arises as a subsequential limit of color representations of $X^{G, \beta, h}$ as $h \rightarrow 0$. Finally, let $S \subseteq[n]$ be such that $|S|$ is odd. Then,

$$
n\left[\sum_{\pi \in \mathcal{B}_{n}} A(S, \pi)\left|T_{S, \pi}\right| \mu(\pi)\right]=\left[\sum_{i \in[n]} \sum_{\pi \in \mathcal{B}_{n}}\left|T_{\{i\}, \pi}\right| \mu(\pi)\right] \cdot\left[\sum_{\pi \in \mathcal{B}_{n}} A(S, \pi) \mu(\pi)\right] .
$$

Proof Note first that by combining Theorem 5.1, Remark 5.2 and Lemma 7.3, it follows that for any $T \subseteq[n]$, we have

$$
\sum_{\pi \in \mathcal{B}_{n}} A(T, \pi) \mu(\pi)= \begin{cases}\frac{n \sum_{\sigma \in\{0,1\}^{n}}(2\|\sigma\|-n) \chi_{T}(\sigma) v_{G, \beta, 0}(\sigma)}{\sum_{i \in[n]} \sum_{\sigma \in\{0,1\}^{n}}(2\|\sigma\|-n)\left(2 \sigma_{i}-1\right) v_{G, \beta, 0}(\sigma)} & \text { if }|T| \text { is odd } \\ \sum_{\sigma \in\{0,1\}^{n}} \chi_{T}(\sigma) v_{G, \beta, 0}(\sigma) & \text { if }|T| \text { is even. }\end{cases}
$$

Next, note that for any such set $T \subseteq[n]$, we have

$$
\begin{aligned}
\sum_{\sigma \in\{0,1\}^{n}}(2\|\sigma\|-n) \chi_{T}(\sigma) v_{G, \beta, 0}(\sigma) & =\sum_{\sigma \in\{0,1\}^{n}} \chi_{T}(\sigma) \sum_{i \in[n]}\left(2 \sigma_{i}-1\right) v_{G, \beta, 0}(\sigma) \\
& =\sum_{i \in[n]} \sum_{\sigma \in\{0,1\}^{n}} \chi_{T \triangle\{i\}}(\sigma) v_{G, \beta, 0}(\sigma) .
\end{aligned}
$$

Since $|S|$ is odd, $|S \triangle\{i\}|$ is even for each $i \in[n]$, and hence, by (18), we have

$$
\sum_{i \in[n]} \sum_{\sigma \in\{0,1\}^{n}} \chi_{S \triangle\{i\}}(\sigma) v_{G, \beta, 0}(\sigma)=\sum_{i \in[n]} \sum_{\pi \in \mathcal{B}_{n}} A(S \triangle\{i\}, \pi) \mu(\pi)
$$


If $A(S, \pi)=1$ for some $\pi \in \mathcal{B}_{n}$, then since $|S|$ is odd, $\pi$ has exactly one odd-sized partition element, which is equal to the restriction of the partition element $T_{S, \pi}$ of $\pi$ to $S$. Further, since $|S \triangle\{j\}|$ is even for each $j \in[n], A(S \triangle\{j\}, \pi)=1$ if and only if $j \in T_{S, \pi}$, and hence

$$
\sum_{\pi \in \mathcal{B}_{n}} \sum_{j \in[n]} A(S \triangle\{j\}, \pi) \mu(\pi)=\sum_{\pi \in \mathcal{B}_{n}} A(S, \pi)\left|T_{S, \pi}\right| \mu(\pi)
$$

Combining the three previous inline equations, we obtain

$$
\sum_{\sigma \in\{0,1\}^{n}}(2\|\sigma\|-n) \chi_{S}(\sigma) v_{G, \beta, 0}(\sigma)=\sum_{\pi \in \mathcal{B}_{n}} A(S, \pi)\left|T_{S, \pi}\right| \mu(\pi) .
$$

Plugging this into (18), and recalling that $|S|$ is odd by assumption, we get

$$
\begin{aligned}
\sum_{\pi \in \mathcal{B}_{n}} A(S, \pi) \mu(\pi) & =\frac{n \sum_{\sigma \in\{0,1\}^{n}}(2\|\sigma\|-n) \chi_{S}(\sigma) v_{G, \beta, 0}(\sigma)}{\sum_{i \in[n]} \sum_{\sigma \in\{0,1\}^{n}}(2\|\sigma\|-n)\left(2 \sigma_{i}-1\right) v_{G, \beta, 0}(\sigma)} \\
& =\frac{n \sum_{\pi \in \mathcal{B}_{n}} A(S, \pi)\left|T_{S, \pi}\right| \mu(\pi)}{\sum_{i \in[n]} \sum_{\pi \in \mathcal{B}_{n}}\left|T_{\{i\}, \pi}\right| \mu(\pi)} .
\end{aligned}
$$

Rearranging this equation, we obtain (17), which is the desired conclusion.

The purpose of the next lemma is to provide a simpler way to calculate the sums in (17) when $\mu$ is a random cluster model on a finite graph $G$ by translating such sums into corresponding sums for Bernoulli percolation.

Lemma 5.4 Let $G=(V, E)$ be a connected and vertex transitive graph. Fix some $r \in(0,1)$ and define $\hat{r}=\frac{r}{2-r}$. Further, let $m$ be the length of the shortest cycle in $G$. Then, there is a constant $Z_{G, \hat{r}}^{\prime \prime}$ such that for any function $f: \mathcal{B}_{n} \rightarrow \mathbb{R}$, we have that

$$
\begin{aligned}
& \frac{Z_{G, r}^{\prime}}{Z_{G, \hat{r}}^{\prime \prime}} \sum_{\pi \in \mathcal{B}_{V}} f(\pi) \mu_{G, r}(\pi) \\
& \quad=\sum_{w \in\{0,1\}^{E}} \hat{r}^{\|w\|}(1-\hat{r})^{|E|-\|w\|} f(\pi[w])\left(1+\mathbb{1}\left(\left(V, E_{w}\right) \text { contains a cycle }\right)\right) \\
& \quad+O\left(\hat{r} x^{m+1}\right) .
\end{aligned}
$$

Proof First recall the definition of the random cluster model corresponding to a parameter $q \geq 0$;

$$
\mu_{G, r, q}(\pi)=\frac{1}{Z_{G, r, q}^{\prime}} \sum_{\substack{w \in\{0,1\}^{E}: \\ \pi[w]=\pi}} r^{\|w\|}(1-r)^{|E|-\|w\|} q^{\|\pi[w]\|}, \quad \pi \in \mathcal{B}_{V} .
$$


where $Z_{G, r, q}^{\prime}$ is a normalizing constant ensuring that this is a probability measure. This implies, in particular, that for any $\pi \in \mathcal{B}_{V}$, we have

$$
\begin{aligned}
Z_{G, r, q}^{\prime} \mu_{G, r, q}(\pi)= & \sum_{\substack{w \in\{0,1\}^{E}: \\
\pi[w]=\pi}} r^{\|w\|}(1-r)^{|E|-\|w\|} q^{\|\pi[w]\|} \\
= & \sum_{\substack{w \in\{0,1\}^{E}: \\
\pi[w]=\pi}} r^{\|w\|}(1-r)^{|E|-\|w\|} q^{|V|-\|w\|} \\
& \cdot\left(1+(q-1) \mathbb{1}\left(\left(V, E_{w}\right) \text { contains a cycle }\right)\right)+O\left(r^{m+1}\right) \\
= & (1-r)^{|E|} q^{|V|} \sum_{\substack{w \in\{0,1\}^{E} \\
\pi[w]=\pi}}\left(\frac{r}{q(1-r)}\right)^{\|w\|} \\
& \cdot\left(1+(q-1) \mathbb{1}\left(\left(V, E_{w}\right) \text { contains a cycle }\right)\right)+O\left(r^{m+1}\right) .
\end{aligned}
$$

If we define $\hat{r}=\frac{r}{r+q(1-r)}$, then we obtain

$$
\begin{aligned}
Z_{G, r, q}^{\prime} \mu_{G, r, q}(\pi)= & (1-r)^{|E|} q^{|V|} \sum_{\substack{w \in\{0,1\}^{E}: \\
\pi[w]=\pi}}\left(\frac{\hat{r}}{1-\hat{r}}\right)^{\|w\|} \\
& \cdot\left(1+(q-1) \mathbb{1}\left(\left(V, E_{w}\right) \text { contains a cycle }\right)\right)+O\left(r^{m+1}\right) . \\
= & \frac{(1-r)^{|E|} q^{|V|}}{(1-\hat{r})^{|E|}} \sum_{\substack{w \in\{0,1\}^{E}: \\
\pi[w]=\pi}} \hat{r}^{\|w\|}(1-\hat{r})^{|E|-\|w\|} \\
& \cdot\left(1+(q-1) \mathbb{1}\left(\left(V, E_{w}\right) \text { contains a cycle }\right)\right)+O\left(r^{m+1}\right) .
\end{aligned}
$$

In particular, this implies that for ant function $f: \mathcal{B}_{n} \rightarrow \mathbb{R}$, we have

$$
\begin{aligned}
Z_{G, r, q}^{\prime} \sum_{\pi \in \mathbb{B}_{V}} f(\pi) \mu_{G, r, q}(\pi)= & \frac{(1-r)^{|E|} q^{|V|}}{(1-\hat{r})^{|E|}} \sum_{w \in\{0,1\}^{E}} f(\pi[w]) \hat{r}^{\|w\|}(1-\hat{r})^{|E|-\|w\|} \\
& \cdot\left(1+(q-1) \mathbb{1}\left(\left(V, E_{w}\right) \text { contains a cycle }\right)\right)+O\left(r^{m+1}\right) .
\end{aligned}
$$

If we let $q=2$ the desired conclusion now follows.

We now use the previous lemma, Lemma 5.4, to give a version of Lemma 5.3 for Bernoulli percolation. To this end, for $r \in(0,1)$ and $q \geq 0$, let $\hat{\mu}_{G, r, q}$ be the measure on $\{0,1\}^{E}$ defined by

$$
\hat{\mu}_{G, \hat{r}, 0}(w):=\hat{r}^{\|w\|}(1-\hat{r})^{|E|-\|w\|} q^{\|\pi[w]\|}, \quad w \in\{0,1\}^{E} .
$$

We then have the following lemma. 
Lemma 5.5 Let $n \in \mathbb{N}$ and let $G=(V, E)$ be a connected graph on $n$ vertices. Let $m$ be the length of the shortest cycle in $G$. Further, let $S \subseteq V$ satisfy $|S|=3$ and let $\Delta_{S}^{(m)}$ be the number of length $m$ cycles in $G$ which contain all the vertices in $S$. Assume that $\beta>0$ is such that $\mu_{G, 1-e^{-2 \beta}}$ is a subsequential limit of color representations of $v_{G, \beta, h}$ as $h \rightarrow 0$. Set $\hat{r}=\frac{r}{2-r}$. Then,

$$
\begin{aligned}
& n\left[\sum_{w \in\{0,1\}^{E}} A(S, \pi[w])\left(\left|T_{S, \pi[w]}\right|-1\right) \hat{\mu}_{G, \hat{r}, 0}(w)\right]+n\left[\Delta_{S}^{(m)} \hat{r}^{m} \cdot(m-1)\right] \\
& =\left[\sum_{i \in[n]} \sum_{w \in\{0,1\}^{E}}\left(\left|T_{\{i\}, \pi[w]}\right|-1\right) \hat{\mu}_{G, \hat{r}, 0}(w)\right] \cdot\left[\sum_{w \in\{0,1\}^{E}} A(S, \pi[w]) \hat{\mu}_{G, \hat{r}, 0}(w)\right] \\
& \quad+O\left(\hat{r}^{m+1}\right) .
\end{aligned}
$$

Proof Note first that by Lemma 5.3, we have that

$$
\begin{aligned}
n \cdot\left[\sum_{\pi \in \mathcal{B}_{n}} A(S, \pi)\left|T_{S, \pi}\right| \mu_{G, r}(\pi)\right] \\
\quad=\left[\sum_{i \in[n]} \sum_{\pi \in \mathcal{B}_{n}}\left|T_{\{i\}, \pi}\right| \mu_{G, r}(\pi)\right] \cdot\left[\sum_{\pi \in \mathcal{B}_{n}} A(S, \pi) \mu_{G, r}(\pi)\right]
\end{aligned}
$$

or equivalently,

$$
\begin{aligned}
& {\left[\sum_{w \in\{0,1\}^{n}} n \cdot \hat{\mu}_{G, r, 2}(w)\right] \cdot\left[\sum_{w \in\{0,1\}^{n}} A(S, \pi[w])\left(\left|T_{S, \pi[w]}\right|-1\right) \hat{\mu}_{G, r, 2}(w)\right]} \\
& \quad=\left[\sum_{i \in[n]} \sum_{w \in\{0,1\}^{n}}\left(\left|T_{\{i\}, \pi[w]}\right|-1\right) \hat{\mu}_{G, r, 2}(w)\right] \cdot\left[\sum_{w \in\{0,1\}^{n}} A(S, \pi[w]) \hat{\mu}_{G, r, 2}(w)\right] .
\end{aligned}
$$

By Lemma 5.4, this implies that

$$
\begin{aligned}
{\left[\sum_{w \in\{0,1\}^{E}} n(1+\mathbb{1}(\pi[w] \text { contains a cycle })) \cdot \hat{\mu}_{G, \hat{r}, 0}(w)\right] } \\
\cdot\left[\sum_{w \in\{0,1\}^{E}} A(S, \pi[w])\left(\left|T_{S, \pi[w]}\right|-1\right)(1+\mathbb{1}(\pi[w] \text { contains a cycle })) \hat{\mu}_{G, \hat{r}, 0}(w)\right] \\
=\left[\sum_{i \in[n]} \sum_{w \in\{0,1\}^{E}}\left(\left|T_{\{i\}, \pi[w]}\right|-1\right)(1+\mathbb{1}(\pi[w] \text { contains a cycle })) \hat{\mu}_{G, \hat{r}, 0}(w)\right] \\
\cdot\left[\sum_{w \in\{0,1\}^{E}} A(S, \pi[w])(1+\mathbb{1}(\pi[w] \text { contains a cycle })) \hat{\mu}_{G, \hat{r}, 0}(w)\right]+O\left(\hat{r}^{m+1}\right) .
\end{aligned}
$$


Noting that, by assumption, one needs at least $m$ edges to make a cycle in $G$, it follows that the previous equation is equivalent to that

$$
\begin{aligned}
& n\left[\sum_{w \in\{0,1\}^{E}} A(S, \pi[w])\left(\left|T_{S, \pi[w]}\right|-1\right) \hat{\mu}_{G, \hat{r}, 0}(w)\right]+n\left[\Delta_{S}^{(m)} \hat{r}^{m} \cdot(m-1)\right] \\
& =\left[\sum_{i \in[n]} \sum_{w \in\{0,1\}^{E}}\left(\left|T_{\{i\}, \pi[w]}\right|-1\right) \hat{\mu}_{G, \hat{r}, 0}(w)\right] \cdot\left[\sum_{w \in\{0,1\}^{E}} A(S, \pi[w]) \hat{\mu}_{G, \hat{r}, 0}(w)\right] \\
& \quad+O\left(\hat{r}^{m+1}\right)
\end{aligned}
$$

which is the desired conclusion.

We are now ready to give a proof of Theorem 1.5 .

Proof Fix any $S \subseteq V$ with $|S|=3$. Note that with the notation of Lemma 5.5, we have that $m=3$ and that $\Delta_{S}^{(3)}=1$. Moreover, one easily verifies that

$$
\sum_{w \in\{0,1\}^{E}} A(S, \pi[w]) \hat{\mu}_{G, \hat{r}, 0}(w)=\left(1-(1-\hat{r})^{3}\right)+(1-\hat{r})^{3} \cdot 3(n-3) \hat{r}^{2}+O\left(\hat{r}^{3}\right) .
$$

and that

$$
\begin{aligned}
\sum_{i \in[n]} \sum_{w \in\{0,1\}^{E}}\left(\left|T_{\{i\}, \pi[w]}\right|-1\right) \hat{\mu}_{G, \hat{r}, 0}(w)= & (2-1) \cdot(n-1) \hat{r}(1-\hat{r})^{2(n-2)} \\
& +(3-1) \cdot\left(\begin{array}{c}
n-1 \\
2
\end{array}\right)\left(\begin{array}{l}
3 \\
1
\end{array}\right) \hat{r}^{2}+O\left(\hat{r}^{3}\right) .
\end{aligned}
$$

Moreover, using Table 1, one sees that

$$
\sum_{w \in\{0,1\}^{E}} A(S, \pi[w])\left(\left|T_{S, \pi[w]}\right|-1\right) \hat{\mu}_{G, \hat{r}, 0}(w)=3(n-1) \hat{r}^{2}+2\left(7-12 n+3 n^{2}\right) \hat{r}^{3}+O\left(\hat{r}^{4}\right)
$$

This implies, in particular, that

$$
\begin{aligned}
& {\left[\sum_{w \in\{0,1\}^{E}} A(S, \pi[w])\left(\left|T_{S, \pi[w]}\right|-1\right) \hat{\mu}_{G, \hat{r}, 0}(w)\right]+\left[\Delta_{S}^{(m)} \hat{r}^{m} \cdot(m-1)\right]} \\
& \quad=3(n-1) \hat{r}^{2}+2\left(8-12 n+3 n^{2}\right) \hat{r}^{3}+O\left(\hat{r}^{4}\right)
\end{aligned}
$$

and that

$$
\begin{aligned}
& {\left[\sum_{w \in\{0,1\}^{E}}\left(\left|T_{\{1\}, \pi[w]}\right|-1\right) \hat{\mu}_{G, \hat{r}, 0}(w)\right] \cdot\left[\sum_{w \in\{0,1\}^{E}} A(S, \pi[w]) \hat{\mu}_{G, \hat{r}, 0}(w)\right]+O\left(\hat{r}^{m+1}\right)} \\
& \quad=3(n-1) \hat{r}^{2}+6(n-1)(n-3) \hat{r}^{3}+O\left(\hat{r}^{4}\right)
\end{aligned}
$$


Table 1 The local edge patterns we need to consider (up to permutations) to obtain the expression in (21).

\begin{tabular}{|c|c|c|c|}
\hline & Pattern & $\left|T_{S, \pi[w]}\right|$ & Probability up to $O\left(\hat{r}^{4}\right)$ \\
\hline (i) & & 2 & $3(n-3)(n-4) \hat{r}^{3}$ \\
\hline (ii) & & 4 & $(n-3) \hat{r}^{3}$ \\
\hline (iii) & & 2 & $3(n-3) \hat{r}^{2}(1-\hat{r})^{2(n-2)}$ \\
\hline (iv) & & 3 & $3(n-3)(n-4) \hat{r}^{3}$ \\
\hline (v) & & 3 & $3\left(\begin{array}{c}n-3 \\
2\end{array}\right) \hat{r}^{3}$ \\
\hline (vi) & & 4 & $3 \cdot 2(n-3) \hat{r}^{3}$ \\
\hline (vii) & & 3 & $3 \hat{r}^{2}(1-\hat{r})^{1+3(n-3)}$ \\
\hline (viii) & & 4 & $3 \cdot 2(n-3) \hat{r}^{3}$ \\
\hline (ix) & & 4 & $3(n-3) \hat{r}^{3}$ \\
\hline (x) & & 3 & $\hat{r}^{3}$ \\
\hline
\end{tabular}

and hence (20) does not hold. By Lemma 5.5, this implies the desired conclusion.

\section{Color Representations for large $h$}

In this section we show that for any finite graph $G$ and any $\beta>0, v_{G, \beta, h}$ has a color representation for all sufficiently large $h$. The main idea of the proof is to show that a very specific formal solution to (2), which was first used in [8], is in fact nonnegative, and hence a color representation of $v_{G, \beta, h}$, whenever $h$ is large enough.

Proof of Theorem 1.7 Fix $n \geq 1$ and $\beta>0$.

For $T \subseteq[n]$ with $|T| \geq 2$, recall that we let $\pi[T]$ denote the unique partition $\pi \in \mathcal{B}_{n}$ which is such that $T$ is a partition element of $\pi$, and all other partition elements has size one. Let $\pi[\emptyset]$ denote the unique partition $\pi \in \mathcal{B}_{n}$ in which all partition elements are singletons. Let $p_{h}:=v_{K_{n}, \beta, h}\left(1^{\{1\}}\right)$. Define

$$
\mu(\pi):=
$$




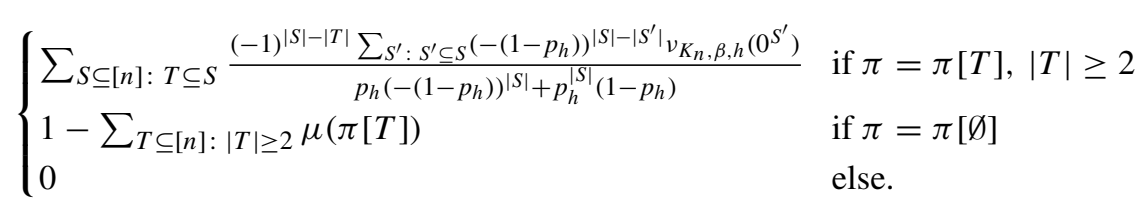

By the proof of Theorem 1.6 in [8], we have $\Phi_{p}^{*}(\mu)=v_{K_{n}, \beta, h}$. We will show that, given the assumptions, $\mu(\pi) \geq 0$ for all $\pi \in \mathcal{B}_{n}$, and hence $\mu \in \Phi_{p}^{-1}\left(v_{K_{n}, \beta, h}\right)$. To this end, note first that for $\sigma \in\{0,1\}^{n}$, we have

$$
v_{K_{n}, \beta, h}(\sigma)=Z_{K_{n}, \beta, h}^{-1} \exp \left(\left(\sqrt{\beta} \sum_{i \in[n]}\left(2 \sigma_{i}-1\right)+h / \sqrt{\beta}\right)^{2} / 2\right)
$$

where $Z_{K_{n}, \beta, h}$ is the corresponding normalizing constant. This implies, in particular, that for any $S \subseteq[n]$,

$$
\lim _{h \rightarrow \infty} \frac{v_{K_{n}, \beta, h}\left(0^{S} 1^{[n] \backslash S}\right)}{v_{K_{n}, \beta, h}\left(1^{[n]}\right)}=\lim _{h \rightarrow \infty} e^{-2|S|(h+\beta(n-|S|))}= \begin{cases}1 & \text { if } S=\emptyset \\ 0 & \text { else. }\end{cases}
$$

This implies, in particular, that $\lim _{h \rightarrow \infty} p_{h}=1$ and that as $h \rightarrow \infty$, we have

$$
Z_{K_{n}, \beta, h} \sim v_{K_{n}, \beta, h}\left(0^{\emptyset} 1^{[n]}\right) \rightarrow 1
$$

Similarly, one shows that for any $S \subseteq[n]$, we have

$$
v_{K_{n}, \beta, h}\left(0^{S}\right) \sim \begin{cases}v_{K_{n}, \beta, h}\left(0^{S} 1^{[n] \backslash S}\right) & \text { if } \beta|S| \leq h \\ v_{K_{n}, \beta, h}\left(0^{[n]} 1^{\emptyset}\right) & \text { if } \beta|S| \geq h\end{cases}
$$

Since $\beta(n-1) \leq h$ by assumption, it follows that $v_{K_{n}, \beta, h}\left(0^{S}\right) \sim v_{K_{n}, \beta, h}\left(0^{S} 1^{[n] \backslash S}\right)$ for all $S \subseteq[n]$, and hence, in particular, that $p_{h} \sim v_{K_{n}, \beta, h}\left(0^{[1]} 1^{[n] \backslash\{1\}}\right)$.

Combining these observations, it follows that for any $S \subseteq[n]$ and any $k \in S$, we have

$$
\begin{aligned}
& \frac{\left(1-p_{h}\right) v_{K_{n}, \beta, h}\left(0^{S \backslash\{k\}}\right)}{v_{K_{n}, \beta, h}\left(0^{S}\right)} \sim \frac{v_{K_{n}, \beta, h}\left(0^{\{1\}} 1^{[n] \backslash\{1\}}\right) v_{K_{n}, \beta, h}\left(0^{S \backslash\{k\}} 1^{[n] \backslash(S \backslash\{k\})}\right)}{v_{K_{n}, \beta, h}\left(0^{\emptyset} 1^{[n]}\right) v_{K_{n}, \beta, h}\left(0^{S} 1^{[n] \backslash S}\right)} \\
& =e^{-4 \beta(|S|-1)}
\end{aligned}
$$

and hence when $h$ is sufficiently large,

$$
v_{K_{n}, \beta, h}\left(0^{S}\right) \sim\left[\prod_{i=1}^{|S|} p e^{4 \beta(|S|-i)}\right] v_{K_{n}, \beta, h}\left(1^{\emptyset}\right)=\left(1-p_{h}\right)^{|S|} e^{2 \beta|S|(|S|-1)}
$$


We will now use the equations above to describe the behavior of (22). To this end, fix some $S \subseteq[n]$ and note that by combining (26) and (25), we obtain

$$
\begin{aligned}
& \sum_{S^{\prime}: S^{\prime} \subseteq S}(-1)^{|S|-\left|S^{\prime}\right|}\left(1-p_{h}\right)^{|S|-\left|S^{\prime}\right|} v_{K_{n}, \beta, h}\left(0^{S^{\prime}}\right) \\
& \quad=(-1)^{|S|}\left(1-p_{h}\right)^{|S|} \sum_{S^{\prime}: S^{\prime} \subseteq S}(-1)^{\left|S^{\prime}\right|} e^{-2 \beta\left|S^{\prime}\right|\left(\left|S^{\prime}\right|-1\right)}+o\left(v_{K_{n}, \beta, h}\left(0^{S}\right)\right) .
\end{aligned}
$$

Using (22), it follows that if $T \subseteq[n]$ and $|T| \geq 2$, then

$$
\begin{aligned}
& \mu(\pi[T])=\sum_{S \subseteq[n]: T \subseteq S} \frac{(-1)^{|S|-|T|} \sum_{S^{\prime}: S^{\prime} \subseteq S}\left(-\left(1-p_{h}\right)\right)^{|S|-\left|S^{\prime}\right|} v_{K_{n}, \beta, h}\left(0^{S^{\prime}}\right)}{p_{h}\left(-\left(1-p_{h}\right)\right)^{|S|}+p_{h}^{|S|}\left(1-p_{h}\right)} \\
& =\sum_{S \subseteq[n]: T \subseteq S} \\
& (-1)^{|S|-|T|}\left[\left(-\left(1-p_{h}\right)\right)^{|S|} \sum_{S^{\prime}: S^{\prime} \subseteq S}(-1)^{\left|S^{\prime}\right|} e^{2 \beta\left|S^{\prime}\right|\left(\left|S^{\prime}\right|-1\right)}+o\left(v_{K_{n}, \beta, h}\left(0^{S}\right)\right)\right] \\
& 1-p_{h}+o\left(1-p_{h}\right) \\
& =\frac{1}{1-p_{h}} \sum_{S \subseteq[n]: T \subseteq S}(-1)^{|T|}\left(1-p_{h}\right)^{|S|} \sum_{S^{\prime}: S^{\prime} \subseteq S}(-1)^{\left|S^{\prime}\right|} e^{2 \beta\left|S^{\prime}\right|\left(\left|S^{\prime}\right|-1\right)} \\
& +o\left(\frac{v_{K_{n}, \beta, h}\left(0^{T}\right)}{1-p_{h}}\right) \text {. }
\end{aligned}
$$

We now rewrite the previous equation. To this end, note first that

$$
\begin{aligned}
& \sum_{S \subseteq[n]: T \subseteq S}\left(1-p_{h}\right)^{|S|} \sum_{S^{\prime}: S^{\prime} \subseteq S}(-1)^{\left|S^{\prime}\right|} e^{2 \beta\left|S^{\prime}\right|\left(\left|S^{\prime}\right|-1\right)} \\
= & \sum_{S^{\prime} \subseteq[n]}(-1)^{\left|S^{\prime}\right|} e^{2 \beta\left|S^{\prime}\right|\left(\left|S^{\prime}\right|-1\right)} \sum_{S \subseteq[n]: T \cup S^{\prime} \subseteq S}\left(1-p_{h}\right)^{|S|} \\
= & \sum_{S^{\prime} \subseteq[n]}(-1)^{\left|S^{\prime}\right|} e^{2 \beta\left|S^{\prime}\right|\left(\left|S^{\prime}\right|-1\right)}\left(1-p_{h}\right)^{\left|T \cup S^{\prime}\right|}\left(2-p_{h}\right)^{n-\left|T \cup S^{\prime}\right|} .
\end{aligned}
$$

Again using (26), it follows that the largest terms in this sum is of order $v_{K_{n}, \beta, h}\left(0^{T}\right)$, and hence, using symmetry, it follows that the previous equation is equal to

$$
\begin{aligned}
& \sum_{S^{\prime} \subseteq[n]:\left|S^{\prime}\right| \leq|T|}(-1)^{\left|S^{\prime}\right|} e^{2 \beta\left|S^{\prime}\right|\left(\left|S^{\prime}\right|-1\right)} \cdot\left(1-p_{h}\right)^{\left|T \cup S^{\prime}\right|}\left(2-p_{h}\right)^{n-\left|T \cup S^{\prime}\right|} \\
& \quad+o\left(v_{K_{n}, \beta, h}\left(0^{T}\right)\right) .
\end{aligned}
$$


Again using that $v_{K_{n}, \beta, h}$ is invariant under permutations, it follows that this is equal to

$$
\begin{aligned}
& \sum_{i=0}^{|T|}(-1)^{i} e^{2 \beta i(i-1)} \sum_{j=0}^{i}\left(\begin{array}{c}
|T| \\
j
\end{array}\right)\left(\begin{array}{c}
n-|T| \\
i-j
\end{array}\right)\left(1-p_{h}\right)^{|T|+(i-j)}(1+p)^{n-(i-j)} \\
& \quad+o\left(v_{K_{n}, \beta, h}\left(0^{T}\right)\right) \\
& \quad=\left(1-p_{h}\right)^{|T|} \sum_{i=0}^{|T|}(-1)^{i} e^{2 \beta i(i-1)}\left(|T|+n\left(1-p_{h}\right)\right)^{i}\left(2-p_{h}\right)^{n-i}+o\left(v_{K_{n}, \beta, h}\left(0^{T}\right)\right)
\end{aligned}
$$

Summing up, we have showed that for any $T \subseteq[n]$ with $|T| \geq 2$, we have that

$$
\begin{aligned}
\mu(\pi[T])= & \left(1-p_{h}\right)^{|T|-1} \sum_{i=0}^{|T|}(-1)^{|T|-i} e^{2 \beta i(i-1)}\left(|T|+n\left(1-p_{h}\right)\right)^{i}\left(2-p_{h}\right)^{n-i} \\
& +o\left(\frac{v_{K_{n}, \beta, h}\left(0^{T}\right)}{1-p_{h}}\right) .
\end{aligned}
$$

Since for any positive and strictly increasing function $f: \mathbb{N} \rightarrow \mathbb{R}$, we have that

$$
\sum_{i=0}^{|T|}(-1)^{|T|-i} e^{i f(i)}>\sum_{i=|T|-1}^{|T|}(-1)^{|T|-i} e^{i f(i)}>0
$$

it follows that

$$
\begin{aligned}
& \sum_{i=0}^{|T|}(-1)^{|T|-i} e^{2 \beta i(i-1)}\left(|T|+n\left(1-p_{h}\right)\right)^{i}\left(2-p_{h}\right)^{n-i} \\
& \geq \sum_{i=|T|-1}^{|T|}(-1)^{|T|-i} e^{2 \beta i(i-1)}\left(|T|+n\left(1-p_{h}\right)\right)^{i}\left(2-p_{h}\right)^{n-i} \\
& =\left(|T|+n\left(1-p_{h}\right)\right)^{|T|-1}\left(2-p_{h}\right)^{n-|T|} e^{2 \beta|T|(|T|-1)} \\
& \quad \cdot\left[\left(|T|+n\left(1-p_{h}\right)\right)-e^{-4 \beta(|T|-1)}\left(2-p_{h}\right)\right] .
\end{aligned}
$$

This is clearly larger than zero, and in fact, by (26), as $h$ tends to infinity, it is asymptotic to

$$
\left(1-p_{h}\right)^{-|T|} v_{K_{n}, \beta, h}\left(0^{T}\right)|T|^{|T|-1}\left[|T|-e^{-4 \beta(|T|-1)}\right] .
$$


This implies that the error term in (27) is much smaller than the rest of the expression, which is strictly positive, i.e.,

$\mu(\pi[T]) \sim\left(1-p_{h}\right)^{|T|-1} \sum_{i=0}^{|T|}(-1)^{|T|-i} e^{2 \beta i(i-1)}\left(|T|+n\left(1-p_{h}\right)\right)^{i}\left(2-p_{h}\right)^{n-i}>0$.

It now remains to show only that $\mu(\pi[\emptyset])>0$. To this end, note first that for any $T \subseteq[n]$ with $|T| \geq 2$, we have that

$$
\begin{aligned}
& \left(1-p_{h}\right)^{|T|-1} \sum_{i=0}^{|T|}(-1)^{|T|-i} e^{2 \beta i(i-1)}\left(|T|+n\left(1-p_{h}\right)\right)^{i}\left(2-p_{h}\right)^{n-i} \\
& \quad \leq\left(1-p_{h}\right)^{-1}(2 n)^{n} \sum_{i=0}^{|T|}\left(1-p_{h}\right)^{|T|-i}\left(1-p_{h}\right)^{i} e^{2 \beta i(i-1)} .
\end{aligned}
$$

By (26), $\left(1-p_{h}\right)^{i} e^{2 \beta i(i-1)} \sim v_{K_{n}, \beta, h}\left(0^{S}\right)$. Since $v_{K_{n}, \beta, h}\left(0^{S}\right)$ tends to zero as $h \rightarrow \infty$ for any $S \subseteq[n]$ with $|S| \geq 1$, it follows that $\lim _{h \rightarrow \infty} \mu(\pi[T])=0$ provided that $\lim _{h \rightarrow \infty} v_{K_{n}, \beta, h}\left(0^{T}\right) /\left(1-p_{h}\right)=0$. To see that this holds, note simply that by (26),

$$
v_{K_{n}, \beta, h}\left(0^{S}\right) /\left(1-p_{h}\right) \sim \frac{v_{K_{n}, \beta, h}\left(0^{T} 1^{[n] \backslash T}\right)}{v_{K_{n}, \beta, h}\left(0^{[1]} 1^{[n] \backslash[1]}\right)}=e^{-2(|T|-1)(|h|+\beta(n-|T|-1))}
$$

which clearly tends to zero as $h \rightarrow \infty$. This concludes the proof.

Remark 6.1 The previous proof shows that the formal solution to (2) given by (22) is a color representation of $v_{K_{n}, \beta, h}$ whenever $\beta$ is fixed and $h$ is sufficiently large. One might hence ask if (22) is also nonnegative for fixed $h \neq 0$ as $\beta \rightarrow 0$. This is, however, not the case, and one can if fact show that for any fixed $h \neq 0$, when $\beta>0$ is sufficiently small, $\mu(\pi[T])<0$ for any $T \subseteq[n]$ which is such that $|T|$ is odd.

Remark 6.2 Given that the relationship we get between $\beta$ and $h$ in Theorem 1.7 is quite far from the conjectured result, one might try to get a stronger result by optimizing the above proof. However, using Mathematica, one can check that the particular formal solution to (2) given by (22) in fact not nonnegative when $(n-1) \beta>h$ when $n=3,4,5$.

\section{Technical Lemmas}

In this section we collect and prove the technical lemmas which have been used throughout the paper.

Lemma 7.1 Let $n \in \mathbb{N}$ and let $G=(V, E)$ be a graph with $n$ vertices. Further, let $\beta>0$ be fixed and, for $h \geq 0$, define $p_{G, \beta}(h):=v_{G, \beta, h}\left(1^{\{1\}}\right)$. Finally, let $\sigma \in\{0,1\}^{n}$. 
Then,

$$
\begin{aligned}
\left.\frac{\mathrm{d} v_{G, \beta, h}(\sigma)}{d p_{G, \beta}}\right|_{h=0} & =\frac{2(2\|\sigma\|-n) v_{G, \beta, 0}(\sigma)}{\sum_{\hat{\sigma} \in\{0,1\}^{n}}\left(2 \hat{\sigma}_{1}-1\right)(2\|\hat{\sigma}\|-n) v_{G, \beta, 0}(\hat{\sigma})} \\
& =\frac{2 n(2\|\sigma\|-n) v_{G, \beta, 0}(\sigma)}{\sum_{\hat{\sigma} \in\{-1,1\}^{n}}(2\|\hat{\sigma}\|-n)^{2} v_{G, \beta, 0}(\hat{\sigma})} .
\end{aligned}
$$

Proof By definition, for any $h \geq 0$ we have

$$
v_{G, \beta, h}(\sigma)=Z_{G, \beta, h}^{-1} \exp \left(\beta \sum_{\{i, j\} \in E}\left(2 \sigma_{i}-1\right)\left(2 \sigma_{j}-1\right)+h(2\|\sigma\|-n)\right),
$$

where

$$
Z_{G, \beta, h}=\sum_{\hat{\sigma} \in\{0,1\}^{n}} \exp \left(\beta \sum_{\{i, j\} \in E}\left(2 \hat{\sigma}_{i}-1\right)\left(2 \hat{\sigma}_{j}-1\right)+h(2\|\hat{\sigma}\|-n)\right) .
$$

If we differentiate $Z_{G, \beta, h} v_{G, \beta, h}(\sigma)$ with respect to $h$, we get

$$
\left.\frac{\mathrm{d}\left(Z_{G, \beta, h} v_{G, \beta, h}(\sigma)\right)}{d h}\right|_{h=0}=(2\|\sigma\|-n) Z_{G, \beta, 0} v_{G, \beta, 0}(\sigma) .
$$

From this it follows that

$$
\begin{aligned}
\left.\frac{\mathrm{d} Z_{G, \beta, h}}{d h}\right|_{h=0} & =\left.\sum_{\hat{\sigma} \in\{0,1\}^{n}} \frac{\mathrm{d}\left(Z_{G, \beta, h} v_{G, \beta, h}(\hat{\sigma})\right)}{d h}\right|_{h=0} \\
& =\sum_{\hat{\sigma} \in\{0,1\}^{n}}(2\|\hat{\sigma}\|-n) Z_{G, \beta, 0} v_{G, \beta, 0}(\hat{\sigma}) \\
& \left.=Z_{G, \beta, 0} \mathbb{E}\left[2\left\|X^{G, \beta, 0}\right\|-n\right)\right]=Z_{G, \beta, 0} \cdot 0=0
\end{aligned}
$$

As

$$
\frac{\mathrm{d}\left(Z_{G, \beta, h} v_{G, \beta, h}(\sigma)\right)}{d h}=\frac{\mathrm{d} Z_{G, \beta, h}}{d h} v_{G, \beta, h}(\sigma)+Z_{G, \beta, h} \frac{\mathrm{d} v_{G, \beta, h}(\sigma)}{d h}
$$

it follows that

$$
\left.\frac{\mathrm{d} v_{G, \beta, h}(\sigma)}{d h}\right|_{h=0}=(2\|\sigma\|-n) v_{G, \beta, 0}(\sigma)
$$

and hence

$$
\left.\frac{\mathrm{d} p_{G, \beta}}{d h}\right|_{h=0}=\sum_{\substack{\hat{\sigma} \in\{0,1\}^{n}: \\ \hat{\sigma}_{1}=1}}(2\|\hat{\sigma}\|-n) v_{G, \beta, 0}(\hat{\sigma})
$$


Combining the two previous equations, we obtain

$$
\left.\frac{\mathrm{d} v_{G, \beta, h}(\sigma)}{d p_{G, \beta}}\right|_{h=0}=\left.\frac{\mathrm{d} v_{G, \beta, h}(\sigma)}{d h}\left(\frac{\mathrm{d} p_{G, \beta}}{d h}\right)^{-1}\right|_{h=0}=\frac{(2\|\sigma\|-n) v_{G, \beta, 0}(\sigma)}{\sum_{\hat{\sigma} \in\{0,1\}^{n}: \hat{\sigma}_{1}=1}(2\|\hat{\sigma}\|-n) v_{G, \beta, 0}(\hat{\sigma})}
$$

The desired conclusion now follows by noting that

$$
\begin{aligned}
\sum_{\hat{\sigma} \in\{0,1\}^{n}: \hat{\sigma}_{1}=1}(2\|\hat{\sigma}\|-n) v_{G, \beta, 0}(\hat{\sigma}) & =\frac{1}{2} \sum_{\hat{\sigma} \in\{0,1\}^{n}}\left(2 \hat{\sigma}_{1}-1\right)(2\|\hat{\sigma}\|-n) v_{G, \beta, 0}(\hat{\sigma}) \\
& =\frac{1}{2 n} \sum_{\hat{\sigma} \in\{0,1\}^{n}}(2\|\hat{\sigma}\|-n)^{2} v_{G, \beta, 0}(\hat{\sigma}) .
\end{aligned}
$$

Lemma 7.2 Let $n \in \mathbb{N}$. Further, let $v \in \mathcal{P}\left(\{0,1\}^{n}\right)$ and let $S \subseteq[n]$. Then,

$$
\sum_{T: T \subseteq S} v\left(1^{T}\right)(-2)^{|T|}=\sum_{T: T \subseteq S}(-1)^{|T|} v\left(1^{T} 0^{S \backslash T}\right)
$$

\section{Proof}

$$
\begin{aligned}
\sum_{S^{\prime}: S^{\prime} \subseteq S} v\left(1^{S^{\prime}}\right)(-2)^{\left|S^{\prime}\right|} & =\sum_{S^{\prime}: S^{\prime} \subseteq S} \sum_{T: T \subseteq S^{\prime}} v\left(1^{S^{\prime}}\right)(-1)^{\left|S^{\prime}\right|} \\
& =\sum_{T: T \subseteq S} \sum_{S^{\prime}: T \subseteq S^{\prime} \subseteq S} v\left(1^{S^{\prime}}\right)(-1)^{\left|S^{\prime}\right|} \\
& =\sum_{T: T \subseteq S} \sum_{S^{\prime \prime}: S^{\prime \prime} \subseteq S \backslash T} v\left(1^{T \cup S^{\prime \prime}}\right)(-1)^{\left|T \cup S^{\prime \prime}\right|} \\
& =\sum_{T: T \subseteq S}(-1)^{|T|} \sum_{S^{\prime \prime}: S^{\prime \prime} \subseteq S \backslash T} v\left(1^{T \cup S^{\prime \prime}}\right)(-1)^{\left|S^{\prime \prime}\right|} \\
& =\sum_{T: T \subseteq S}(-1)^{|T|} v\left(1^{T} 0^{S \backslash T}\right) .
\end{aligned}
$$

Lemma 7.3 Let $n \in \mathbb{N}$ and let $G$ be a graph with $n$ vertices. Further, let $\beta>0$ be fixed and, for $h \geq 0$, define $p_{G, \beta}(h):=v_{G, \beta, h}\left(1^{\{1\}}\right)$. Finally, let $S \subseteq[n]$. Then, the following two equations hold.

(i)

$$
\sum_{S^{\prime} \subseteq S}(-2)^{|S|} v_{G, \beta, 0}\left(1^{S^{\prime}}\right)=(-1)^{|S|} \sum_{\sigma \in\{0,1\}^{n}} \chi_{S}(\sigma) v_{G, \beta, 0}(\sigma)
$$


(ii)

$$
\begin{aligned}
& \left.\sum_{S^{\prime} \subseteq S}(-2)^{|S|-1} \frac{\mathrm{d} v_{G, \beta, h}\left(1^{S^{\prime}}\right)}{d p_{G, \beta}}\right|_{h=0} \\
& =n(-1)^{|S|+1} \frac{\sum_{\sigma \in\{0,1\}^{n}}(2\|\sigma\|-n) \chi_{S}(\sigma) v_{G, \beta, 0}(\sigma)}{\sum_{\sigma \in\{0,1\}^{n}}(2\|\sigma\|-n)^{2} v_{G, \beta, 0}(\sigma)} .
\end{aligned}
$$

Proof By Lemma 7.2, we have that

$$
\begin{aligned}
& \sum_{T: T \subseteq S}(-2)^{|T|} v_{G, \beta, 0}\left(1^{T}\right)=\sum_{T: T \subseteq S}(-1)^{|T|} v_{G, \beta, 0}\left(1^{T} 0^{S \backslash T}\right) \\
& =\sum_{T: T \subseteq[n]}(-1)^{|T \cap S|} v_{G, \beta, 0}\left(1^{T} 0^{[n] \backslash T}\right)=\sum_{\sigma \in\{0,1\}^{n}} \prod_{i \in S}\left(-\left(2 \sigma_{i}-1\right)\right) v_{G, \beta, 0}(\sigma) \\
& =(-1)^{|S|} \sum_{\sigma \in\{0,1\}^{n}} \prod_{i \in S}\left(2 \sigma_{i}-1\right) v_{G, \beta, 0}(\sigma)=(-1)^{|S|} \sum_{\sigma \in\{0,1\}^{n}} \chi_{S}(\sigma) v_{G, \beta, 0}(\sigma)
\end{aligned}
$$

and hence (i) holds. To see that (ii) holds, note first that by the same argument as above, it follows that

$$
\left.\sum_{T: T \subseteq S}(-2)^{|T|-1} \frac{\mathrm{d} v_{G, \beta, h}\left(1^{T}\right)}{d p_{G, \beta}}\right|_{h=0}=\left.2^{-1}(-1)^{|S|+1} \sum_{\sigma \in\{0,1\}^{n}} \chi_{S}(\sigma) \frac{\mathrm{d} v_{G, \beta, h}(\sigma)}{d p_{G, \beta}}\right|_{h=0} .
$$

Applying Lemma 7.1, we obtain (ii).

Lemma 7.4 Let $n \in \mathbb{N}, \beta>0$ and $h \geq 0$. Then,

$$
\begin{aligned}
& \sum_{\sigma \in\{0,1\}^{n}} \chi_{\emptyset}(\sigma) v_{K_{n}, \beta, h}(\sigma)=1 \\
& n \sum_{\sigma \in\{0,1\}^{n}}(2\|\sigma\|-1) \chi_{\{1\}}(\sigma) v_{K_{n}, \beta, h}(\sigma)=\sum_{\sigma \in\{0,1\}^{n}}(2\|\sigma\|-n)^{2} v_{K_{n}, \beta, h}(\sigma) \\
& (n)_{2} \sum_{\sigma \in\{0,1\}^{n}} \chi_{[2]}(\sigma) v_{K_{n}, \beta, h}(\sigma)=\sum_{\sigma \in\{0,1\}^{n}}(2\|\sigma\|-n)^{2} v_{K_{n}, \beta, h}(\sigma)-n \\
& (n)_{3} \sum_{\sigma \in\{0,1\}^{n}}(2\|\sigma\|-n) \chi_{[3]}(\sigma) v_{K_{n}, \beta, h}(\sigma) \\
& \quad=\sum_{\sigma \in\{0,1\}^{n}}(2\|\sigma\|-n)^{4} v_{K_{n}, \beta, h}(\sigma)-(3 n-2) \sum_{\sigma \in\{0,1\}^{n}}(2\|\sigma\|-n)^{2} v_{K_{n}, \beta, h}(\sigma)
\end{aligned}
$$




$$
\begin{aligned}
(n)_{4} & \sum_{\sigma \in\{0,1\}^{n}} \chi_{[4]}(\sigma) v_{K_{n}, \beta, h}(\sigma) \\
= & \sum_{\sigma \in\{0,1\}^{n}}(2\|\sigma\|-n)^{4} v_{K_{n}, \beta, h}(\sigma)-2(3 n-4) \sum_{\sigma \in\{0,1\}^{n}}(2\|\sigma\|-n)^{2} v_{K_{n}, \beta, h}(\sigma) \\
& +3\left(n^{2}-2 n\right)
\end{aligned}
$$

and

$$
\begin{aligned}
(n)_{5} & \sum_{\sigma \in\{0,1\}^{n}}(2\|\sigma\|-n) \chi_{[5]}(\sigma) v_{K_{n}, \beta, h}(\sigma) \\
= & \sum_{\sigma \in\{0,1\}^{n}}(2\|\sigma\|-n)^{6} v_{K_{n}, \beta, h}(\sigma)-10(n-2) \sum_{\sigma \in\{0,1\}^{n}}(2\|\sigma\|-n)^{4} v_{K_{n}, \beta, h}(\sigma) \\
& +\left(15 n^{2}-50 n+24\right) \sum_{\sigma \in\{0,1\}^{n}}(2\|\sigma\|-n)^{2} v_{K_{n}, \beta, h}(\sigma) .
\end{aligned}
$$

Proof By symmetry, for each $m \in[n]$ we have that

$$
\begin{aligned}
\left(\begin{array}{l}
n \\
m
\end{array}\right) \sum_{\sigma \in\{0,1\}^{n}} \chi_{[m]}(\sigma) v_{K_{n}, \beta, h}(\sigma) & =\sum_{\substack{S \subseteq[n]: \\
|S|=m}} \sum_{\sigma \in\{0,1\}^{n}} \chi_{S}(\sigma) v_{K_{n}, \beta, h}(\sigma) \\
& =\sum_{\sigma \in\{0,1\}^{n}} v_{K_{n}, \beta, h}(\sigma) \sum_{\substack{S \subseteq[n]: \\
|S|=m}} \chi_{S}(\sigma)
\end{aligned}
$$

and, completely analogously,

$$
\left(\begin{array}{l}
n \\
m
\end{array}\right) \sum_{\sigma \in\{0,1\}^{n}}(2\|\sigma\|-n) \chi_{[m]}(\sigma) v_{K_{n}, \beta, h}(\sigma)=\sum_{\sigma \in\{0,1\}^{n}}(2\|\sigma\|-n) v_{K_{n}, \beta, h}(\sigma) \sum_{\substack{S \subseteq[n]: \\
|S|=m}} \chi_{S}(\sigma) .
$$

Now fix some $\sigma \in\{0,1\}^{n}$. Then, we have that

$$
\sum_{i \in[n]} \mathbb{1}_{\sigma_{i}=1}=\|\sigma\|
$$

and hence

$$
\sum_{S \subseteq[n]:|S|=m} \chi_{S}(\sigma)=\sum_{i=0}^{n-\|\sigma\|}\left(\begin{array}{c}
n-\|\sigma\| \\
i
\end{array}\right)\left(\begin{array}{c}
\|\sigma\| \\
m-i
\end{array}\right)(-1)^{i}
$$

When $m \in$ [5], it follows that 


$$
\begin{aligned}
& \sum_{S \subseteq[n]:|S|=m} \chi_{S}(\sigma) \\
& = \begin{cases}\|\sigma\| & \text { if } m=1 \\
\frac{(2\|\sigma\|-n)^{2}}{2 !}-\frac{n}{2} & \text { if } m=2 \\
\frac{(2\|\sigma\|-n)^{3}}{3 !}-\frac{(3 n-2) \cdot(2\|\sigma\|-n)}{6} & \text { if } m=3 \\
\frac{(2\|\sigma\|-n)^{4}}{4 !}-\frac{(3 n-4) \cdot(2\|\sigma\|-n)^{2}}{12}+\frac{n^{2}-2 n}{8} & \text { if } m=4 \\
\frac{(2\|\sigma\|-n)^{5}}{5 !}-\frac{(n-2) \cdot(2\|\sigma\|-n)^{3}}{12}+\frac{\left(15 n^{2}-50 n+24\right) \cdot(2\|\sigma\|-n)}{120} & \text { if } m=5 .\end{cases}
\end{aligned}
$$

By plugging these expressions into Eqs. (28) and (29), the desired conclusion follows.

Acknowledgements The author acknowledges support from the European Research Council, Grant No. 682537, from Stiftelsen G S Magnusons fond and from Knut and Alice Wallenbergs stiftelse. The author would like to thank Jeffrey E. Steif for many interesting conversation, and in particular for mentioning the example given in Proposition 1.3. The author would also like to thank the anonymous referee for making several useful comments.

Funding Open access funding provided by Royal Institute of Technology.

Open Access This article is licensed under a Creative Commons Attribution 4.0 International License, which permits use, sharing, adaptation, distribution and reproduction in any medium or format, as long as you give appropriate credit to the original author(s) and the source, provide a link to the Creative Commons licence, and indicate if changes were made. The images or other third party material in this article are included in the article's Creative Commons licence, unless indicated otherwise in a credit line to the material. If material is not included in the article's Creative Commons licence and your intended use is not permitted by statutory regulation or exceeds the permitted use, you will need to obtain permission directly from the copyright holder. To view a copy of this licence, visit http://creativecommons.org/licenses/by/4.0/.

\section{References}

1. Aizenman, M., Chayes, J.T., Chayes, L., Newman, C.M.: Discontinuity of the magnetization in the one-dimensional $1 /|x-y|^{2}$ Ising and Potts models. J. Stat. Phys. 50, 1-40 (1988)

2. Bálint, A.: Gibbsianness and non-Gibbsianness in divide and color models. Ann. Prob. 38(4), 16091638 (2010)

3. Bálint, A., Beffara, V., Tassion, V.: Confidence intervals for the critical value in the divide and color model. ALEA Latin Am. J. Prob. Math. Stat. 10(2), 667-679 (2013)

4. Bálint, A., Camia, F., Meester, R.M.J.: Sharp phase transitions and critical behaviour in 2D divide and colour models. Stoch. Process. Appl. 119, 937-965 (2009)

5. Biskup, M., Borgs, C., Chayes, J.T., Kotecky, R.: Gibbs states of graphical representations of the Potts model with external fields. J. Math. Phys. 41, 1170-1210 (2000)

6. Borgs, C., Chayes, J.T.: The covariance matrix of the Potts model: a random cluster analysis. J. Stat. Phys. 82, 1235-1297 (1996)

7. Ellis, R.: Entropy, Large Deviations, and Statistical Mechanics. Springer, New York (1985)

8. Forsström, M.P., Steif, J.E.: An analysis of the induced linear operators associated to divide and color models. J. Theor. Probab. (2020). https://doi.org/10.1007/s10959-020-01001-4

9. Garet, O.: Limit theorems for the painting of graphs by clusters. ESAIM Probab Stat. 5, 105-118 (2001)

10. Grimmett, G.: The Random-Cluster Model. Springer, Berlin (2006)

11. Häggstöm, O.: Coloring percolation clusters at random. Stoch. Process. Appl. 96(2), 213-242 (2001) 
12. Steif, J.E., Tykesson, J.: Generalized divide and color models. ALEA Latin Am. J. Probab. Math. Stat. 16, 899-955 (2019)

Publisher's Note Springer Nature remains neutral with regard to jurisdictional claims in published maps and institutional affiliations. 